\title{
SPECTRAL MULTIPLIERS ON HEISENBERG-REITER AND RELATED GROUPS
}

\author{
ALESSIO MARTINI
}

\begin{abstract}
Let $L$ be a homogeneous sublaplacian on a 2 -step stratified Lie group $G$ of topological dimension $d$ and homogeneous dimension $Q$. By a theorem due to Christ and to Mauceri and Meda, an operator of the form $F(L)$ is bounded on $L^{p}$ for $1<p<\infty$ if $F$ satisfies a scale-invariant smoothness condition of order $s>Q / 2$. Under suitable assumptions on $G$ and $L$, here we show that a smoothness condition of order $s>d / 2$ is sufficient. This extends to a larger class of 2-step groups the results for the Heisenberg and related groups by Müller and Stein and by Hebisch, and for the free group $N_{3,2}$ by Müller and the author.
\end{abstract}

\section{INTRODUCTION}

Let $L$ be a homogeneous sublaplacian on a stratified Lie group $G$ of homogeneous dimension $Q$. Since $L$ is a positive selfadjoint operator on $L^{2}(G)$, a functional calculus for $L$ is defined via the spectral theorem and, for all Borel functions $F$ : $\mathbb{R} \rightarrow \mathbb{C}$, the operator $F(L)$ is bounded on $L^{2}(G)$ whenever the "spectral multiplier" $F$ is bounded. As for the $L^{p}$-boundedness for $p \neq 2$ of $F(L)$, a sufficient condition in terms of smoothness properties of the multiplier $F$ is given by a theorem of Mihlin-Hörmander type due to Christ $[3$ and Mauceri and Meda [16: the operator $F(L)$ is of weak type $(1,1)$ and bounded on $L^{p}(G)$ for all $\left.p \in\right] 1, \infty[$ whenever

$$
\|F\|_{M W_{2}^{s}}:=\sup _{t>0}\|F(t \cdot) \eta\|_{W_{2}^{s}}<\infty
$$

for some $s>Q / 2$, where $W_{2}^{s}(\mathbb{R})$ is the $L^{2}$ Sobolev space of fractional order $s$ and $\eta \in C_{c}^{\infty}(] 0, \infty[)$ is a nontrivial auxiliary function.

A natural question that arises is if the smoothness condition $s>Q / 2$ is sharp. This is clearly true when $G$ is abelian, so $Q$ coincides with the topological dimension $d$ of $G$, and $L$ is essentially the Laplace operator on $\mathbb{R}^{d}$. Take however the smallest nonabelian example of a stratified group, that is, the Heisenberg group $\mathrm{H}_{1}$, which is defined by endowing $\mathbb{R} \times \mathbb{R} \times \mathbb{R}$ with the group law

$$
(x, y, u) \cdot\left(x^{\prime}, y^{\prime}, u^{\prime}\right)=\left(x+x^{\prime}, y+y^{\prime}, u+u^{\prime}+\left(x y^{\prime}-x^{\prime} y\right) / 2\right)
$$

and with the automorphic dilations

$$
\delta_{t}(x, y, u)=\left(t x, t y, t^{2} u\right) .
$$

$\mathrm{H}_{1}$ is a 2-step stratified group, and the homogeneous dimension of $\mathrm{H}_{1}$ is 4 . Nevertheless, a result by Müller and Stein 17 and Hebisch 8 shows that, for a homogeneous sublaplacian on $\mathrm{H}_{1}$, the smoothness condition on the multiplier can be pushed down to $s>d / 2$, where $d=3$ is the topological dimension of $\mathrm{H}_{1}$ (in [17] it is also proved that the condition $s>d / 2$ is sharp). Such an improvement of the Christ-Mauceri-Meda theorem holds not only for $\mathrm{H}_{1}$, but for the larger class

2010 Mathematics Subject Classification. 43A22, 42B15.

Key words and phrases. nilpotent Lie groups, Heisenberg-Reiter groups, spectral multipliers, sublaplacians, Mihlin-Hörmander multipliers, singular integral operators.

The author gratefully acknowledges the support of the Alexander von Humboldt Foundation. 
of Métivier groups (and for direct products of Métivier and abelian groups), and also for differential operators other than sublaplacians (see, e.g., [9, 12]). However it is still an open question whether, for a general stratified Lie group (or even for a general 2-step stratified group), the homogeneous dimension in the smoothness condition can be replaced by the topological dimension.

The aim of this paper is to extend the class of the 2-step stratified groups and sublaplacians for which the smoothness condition in the multiplier theorem can be pushed down to half the topological dimension.

Take for instance the Heisenberg-Reiter group $\mathrm{H}_{d_{1}, d_{2}}$ (cf. [22]), defined by endowing $\mathbb{R}^{d_{2} \times d_{1}} \times \mathbb{R}^{d_{1}} \times \mathbb{R}^{d_{2}}$ with the group law (1) and the automorphic dilations (2); here however $\mathbb{R}^{d_{2} \times d_{1}}$ is the set of the real $d_{2} \times d_{1}$ matrices, and the products $x y^{\prime}, x^{\prime} y$ in (11) are interpreted in the sense of matrix multiplication. $\mathrm{H}_{d_{1}, d_{2}}$ is a 2step stratified group of homogeneous dimension $Q=d_{1} d_{2}+d_{1}+2 d_{2}$ and topological dimension $d=d_{1} d_{2}+d_{1}+d_{2}$. Despite the formal similarity with $\mathrm{H}_{1}$, the group $\mathrm{H}_{d_{1}, d_{2}}$ does not fall into the class of Métivier groups, unless $d_{2}=1$ (in fact, $\mathrm{H}_{d_{1}, 1}$ is the $\left(2 d_{1}+1\right)$-dimensional Heisenberg group $\left.\mathrm{H}_{d_{1}}\right)$. Nevertheless, the technique presented here allows one to handle the case $d_{2}>1$ too.

Namely, let $X_{1,1}, \ldots, X_{d_{2}, d_{1}}, Y_{1}, \ldots, Y_{d_{1}}, U_{1}, \ldots, U_{d_{2}}$ be the left-invariant vector fields on $\mathrm{H}_{d_{1}, d_{2}}$ extending the standard basis of $\mathbb{R}^{d_{2} \times d_{1}} \times \mathbb{R}^{d_{1}} \times \mathbb{R}^{d_{2}}$ at the identity, and define the homogeneous sublaplacian $L$ by

$$
L=-\sum_{j=1}^{d_{1}} \sum_{k=1}^{d_{2}} X_{k, j}^{2}-\sum_{j=1}^{d_{1}} Y_{j}^{2} .
$$

Then a particular instance of our main result reads as follows.

Theorem 1. Suppose that a function $F: \mathbb{R} \rightarrow \mathbb{C}$ satisfies

$$
\|F\|_{M W_{2}^{s}}<\infty
$$

for some $s>d / 2$. Then the operator $F(L)$ is of weak type $(1,1)$ and bounded on $L^{p}\left(\mathrm{H}_{d_{1}, d_{2}}\right)$ for all $\left.p \in\right] 1, \infty[$.

To the best of our knowledge, this result is new, at least in the case $d_{2}>d_{1}$. In fact, in the case $d_{2} \leq d_{1}$, the extension described in [12] of the technique of [8, 9 ] would give the same result. However the technique presented here is different, and yields the result irrespective of the parameters $d_{1}, d_{2}$.

The left quotient of $\mathrm{H}_{d_{1}, d_{2}}$ by the subgroup $\mathbb{R}^{d_{2} \times d_{1}} \times\{0\} \times\{0\}$ gives a homogeneous space diffeomorphic to $\mathbb{R}^{d_{1}} \times \mathbb{R}^{d_{2}}$, and the sublaplacian $L$ corresponds in the quotient to a Grushin operator. In recent joint works with Adam Sikora [15] and Detlef Müller [14, we proved for these Grushin operators on $\mathbb{R}^{d_{1}} \times \mathbb{R}^{d_{2}}$ a sharp spectral multiplier theorem of Mihlin-Hörmander type, where the smoothness requirement is again half the topological dimension of the ambient space.

The proofs in 15, 14 rely heavily on properties of the eigenfunction expansions for the Hermite operators. Since a homogeneous sublaplacian on a 2-step stratified group reduces to a Hermite operator in almost all irreducible unitary representations of the group, it is conceivable that an adaptation of the methods of [15, 14] may give an improvement to the multiplier theorem for 2-step stratified groups, even outside of the Métivier setting. A first result in this direction is shown in 13, where the free 2-step nilpotent Lie group $N_{3,2}$ on three generators is considered, and properties of Laguerre polynomials are exploited (somehow in the spirit of [4, 17, 19]). The argument presented here refines and extends the one in [13.

Theorem 1 above is just a particular case of the result presented here, and we refer the reader to the next section for a precise statement. We remark that the analogue of Theorem 1 holds on $\mathrm{H}_{d_{1}, d_{2}}$ when the sublaplacian $L$ has the more 
general form

$$
L=-\sum_{j=1}^{d_{1}} \sum_{k, k^{\prime}=0}^{d_{2}} a_{k, k^{\prime}}^{j} X_{k, j} X_{k^{\prime}, j}
$$

where $X_{0, j}=Y_{j}$ and $\left(a_{k, k^{\prime}}^{j}\right)_{k, k^{\prime}=0, \ldots, d_{2}}$ is a positive-definite symmetric matrix for all $j \in\left\{1, \ldots, d_{1}\right\}$. Other groups can be considered too, e.g., the complexification of a Heisenberg-Reiter group, or the quotient of the direct product of $\mathrm{H}_{1,3}$ and $N_{3,2}$ given by identifying the respective centers.

\section{The GENERAL SETTING}

Let $G$ be a connected, simply connected nilpotent Lie group of step 2. Recall that, via exponential coordinates, $G$ may be identified with its Lie algebra $\mathfrak{g}$, that is, the tangent space of $G$ at the identity. In turn, $\mathfrak{g}$ may be identified with the Lie algebra of left-invariant vector fields on $G$. We refer to [6] for the basic definitions and further details.

Let $\mathfrak{g}$ be decomposed as $\mathfrak{v} \oplus \mathfrak{z}$, where $\mathfrak{z}$ is the center of $\mathfrak{g}$, and let $\langle\cdot, \cdot\rangle$ be an inner product on $\mathfrak{v}$. The sublaplacian $L$ associated with the inner product is defined by $L=-\sum_{j} X_{j}^{2}$, where $\left\{X_{j}\right\}_{j}$ is any orthonormal basis of $\mathfrak{v}$. Note that, vice versa, by the Poincaré-Birkhoff-Witt theorem, any second-order operator $L$ of the form $-\sum_{j} X_{j}^{2}$ for some basis $\left\{X_{j}\right\}_{j}$ of $\mathfrak{g}$ modulo $\mathfrak{z}$ determines uniquely a linear complement $\mathfrak{v}=\operatorname{span}\left\{X_{j}\right\}_{j}$ of $\mathfrak{z}$ and an inner product on $\mathfrak{v}$ such that $\left\{X_{j}\right\}_{j}$ is orthonormal.

Let $\mathfrak{z}^{*}$ be the dual of $\mathfrak{z}$ and, for all $\eta \in \mathfrak{z}^{*}$, define $J_{\eta}$ as the linear endomorphism of $\mathfrak{v}$ such that $\eta\left(\left[z, z^{\prime}\right]\right)=\left\langle J_{\eta} z, z^{\prime}\right\rangle$ for all $z, z^{\prime} \in \mathfrak{v}$. Clearly $J_{\eta}$ is skewadjoint with respect to the inner product, hence $J_{\eta}^{2}$ is selfadjoint and negative semidefinite, with even rank, for all $\eta \in \mathfrak{z}^{*}$. Set moreover $\dot{\mathfrak{z}}=\mathfrak{z}^{*} \backslash\{0\}$.

Assumption (A). There exist integers $r_{1}, \ldots, r_{d_{1}}>0$ and an orthogonal decomposition $\mathfrak{v}=\mathfrak{v}_{1} \oplus \cdots \oplus \mathfrak{v}_{d_{1}}$ such that, if $P_{1}, \ldots, P_{d_{1}}$ are the corresponding orthogonal projections, then $J_{\eta} P_{j}=P_{j} J_{\eta}$ and $J_{\eta}^{2} P_{j}$ has rank $2 r_{j}$ and a unique nonzero eigenvalue for all $\eta \in \dot{\mathfrak{z}}$ and all $j \in\left\{1, \ldots, d_{1}\right\}$.

Note that from Assumption (A) it follows that $J_{\eta} \neq 0$ for all $\eta \in \dot{\mathfrak{z}}$. Therefore $[\mathfrak{v}, \mathfrak{v}]=\mathfrak{z}$, that is, the decomposition $\mathfrak{g}=\mathfrak{v} \oplus \mathfrak{z}$ is a stratification of $\mathfrak{g}$, and the sublaplacian $L$ is hypoelliptic.

In fact $J_{\eta}$ has constant rank $2\left(r_{1}+\cdots+r_{k}\right)$ for all $\eta \in \dot{\mathfrak{z}}$. If $J_{\eta}$ is invertible for all $\eta \in \dot{\mathfrak{z}}$, then $G$ is a Métivier group, and if in particular $J_{\eta}^{2}=-|\eta|^{2} \mathrm{id}_{\mathfrak{v}}$ for some inner product norm $|\cdot|$ on $\mathfrak{z}^{*}$, then $G$ is an H-type group. The main novelty of our Assumption (A) is that it allows $J_{\eta}$ to have a nonzero kernel when $\eta \in \dot{\mathfrak{z}}$, although the dimension of the kernel must be constant.

The fact that $J_{\eta}$ has constant rank for $\eta \in \dot{\mathfrak{z}}$ depends only on the algebraic structure of $G$. What depends on the inner product, that is, on the sublaplacian $L$, are the values and multiplicities of the eigenvalues of the $J_{\eta}$. The above Assumption (A) asks for a sort of simultaneous diagonalizability of the $J_{\eta}$.

Under our Assumption (A) on the group $G$ and the sublaplacian $L$, we are able to prove the following multiplier theorem.

Theorem 2. Suppose that a function $F: \mathbb{R} \rightarrow \mathbb{C}$ satisfies

$$
\|F\|_{M W_{2}^{s}}<\infty
$$

for some $s>(\operatorname{dim} G) / 2$. Then the operator $F(L)$ is of weak type $(1,1)$ and bounded on $L^{p}(G)$ for all $\left.p \in\right] 1, \infty[$. 
The previously mentioned Heisenberg-Reiter groups $\mathrm{H}_{d_{1}, d_{2}}$ satisfy Assumption (A), where the inner product is determined by the sublaplacian (3), and the orthogonal decomposition of the first layer is given by the natural isomorphism $\mathbb{R}^{d_{2} \times d_{1}} \times \mathbb{R}^{d_{1}} \cong\left(\mathbb{R}^{d_{2}} \times \mathbb{R}\right)^{d_{1}}$. Other examples are the free 2 -step nilpotent Lie group $N_{3,2}$ on 3 generators, considered in 13 , and its complexification $N_{3,2}^{\mathbb{C}}$. Moreover, if $G_{1}$ and $G_{2}$ satisfy Assumption (A), and their centers have the same dimension, then the quotient of $G_{1} \times G_{2}$ given by any linear identification of the centers satisfy Assumption (A). Note that the direct product $G_{1} \times G_{2}$ itself does not satisfy Assumption (A), but an adaptation of the argument presented here allows one to consider that case too. We postpone to the end of this paper a more detailed discussion of these remarks.

From now on, unless otherwise specified, we assume that $G$ and $L$ are a 2 -step stratified group and a homogeneous sublaplacian on $G$ satisfying Assumption (A). Since $L$ is a left-invariant operator, so is any operator of the form $F(L)$. Let $\mathcal{K}_{F(L)}$ denote the convolution kernel of $F(L)$. As shown, e.g., by [12, Theorem 4.6], the previous theorem is a consequence of the following estimate.

Proposition 3. For all $s>(\operatorname{dim} G) / 2$, there exists a weight $w_{s}: G \rightarrow[1, \infty[$ such that $w_{s}^{-1} \in L^{2}(G)$ and, for all compact sets $K \subseteq \mathbb{R}$ and for all functions $F: \mathbb{R} \rightarrow \mathbb{C}$ with $\operatorname{supp} F \subseteq K$,

$$
\left\|w_{s} \mathcal{K}_{F(L)}\right\|_{2} \leq C_{K, s}\|F\|_{W_{2}^{s}}
$$

in particular

$$
\left\|\mathcal{K}_{F(L)}\right\|_{1} \leq C_{K, s}\|F\|_{W_{2}^{s}} .
$$

The rest of the paper, except for the last section, is devoted to the proof of this estimate.

\section{The Joint FUnCTiOnAL CALCULUS}

Let $d_{2}=\operatorname{dim} \mathfrak{z}$, and let $U_{1}, \ldots, U_{d_{2}}$ be any basis of the center $\mathfrak{z}$. Let moreover the "partial sublaplacian" $L_{j}$ be defined as $L_{j}=-\sum_{l} X_{j, l}^{2}$, where $\left\{X_{j, l}\right\}_{l}$ is any orthonormal basis of $\mathfrak{v}_{j}$, for all $j \in\left\{1, \ldots, d_{1}\right\}$; in particular $L=L_{1}+\cdots+L_{d_{1}}$. Then the left-invariant differential operators

$$
L_{1}, \ldots, L_{d_{1}},-i U_{1}, \ldots,-i U_{d_{2}}
$$

are essentially self-adjoint and commute strongly, hence they admit a joint functional calculus (see, e.g., [1]). Therefore, if $\mathbf{L}$ and $\mathbf{U}$ denote the "vectors of operators" $\left(L_{1}, \ldots, L_{d_{1}}\right)$ and $\left(-i U_{1}, \ldots,-i U_{d_{2}}\right)$, and if we identify $\mathfrak{z}^{*}$ with $\mathbb{R}^{d_{2}}$ via the dual basis of $U_{1}, \ldots, U_{n}$, then, for all bounded Borel functions $H: \mathbb{R}^{d_{1}} \times \mathfrak{z}^{*} \rightarrow \mathbb{C}$, the operator $H(\mathbf{L}, \mathbf{U})$ is defined and bounded on $L^{2}(G)$. Moreover $H(\mathbf{L}, \mathbf{U})$ is leftinvariant, and we can express its convolution kernel $\mathcal{K}_{H(\mathbf{L}, \mathbf{U})}$ in terms of Laguerre functions.

Namely, for all $n, k \in \mathbb{N}$, let

$$
L_{n}^{(k)}(t)=\frac{t^{-k} e^{t}}{n !}\left(\frac{d}{d t}\right)^{n}\left(t^{k+n} e^{-t}\right)
$$

be the $n$-th Laguerre polynomial of type $k$, and define

$$
\mathcal{L}_{n}^{(k)}(t)=(-1)^{n} e^{-t} L_{n}^{(k)}(2 t)
$$

Note that, by Assumption (A), for all $\eta \in \dot{\mathfrak{z}}$ and $j \in\left\{1, \ldots, d_{1}\right\}$,

$$
J_{\eta}^{2} P_{j}=-\left(b_{j}^{\eta}\right)^{2} P_{j}^{\eta}
$$

for some orthogonal projection $P_{j}^{\eta}$ of rank $2 r_{j}$ and some $b_{j}^{\eta}>0$. Set moreover

$$
\bar{P}_{j}^{\eta}=P_{j}-P_{j}^{\eta} .
$$


Modulo reordering the $\mathfrak{v}_{j}$ in the decomposition of $\mathfrak{v}$, we may suppose that there exists $\tilde{d}_{1} \in\left\{0, \ldots, d_{1}\right\}$ such that $\operatorname{dim} \mathfrak{v}_{j}>2 r_{j}$ if $j \leq \tilde{d}_{1}$, and $\operatorname{dim} \mathfrak{v}_{j}=2 r_{j}$ if $j>\tilde{d}_{1}$. In particular, $\bar{P}_{j}^{\eta}=0$ and $P_{j}^{\eta}=P_{j}$ for all $j>\tilde{d}_{1}$ and $\eta \in \dot{\mathfrak{z}}$. We will also use the abbreviations $r=\left(r_{1}, \ldots, r_{d_{1}}\right), \mathbb{R}^{r}=\mathbb{R}^{r_{1}} \times \cdots \times \mathbb{R}^{r_{d_{1}}}, \mathbb{N}^{r}=\mathbb{N}^{r_{1}} \times \cdots \times \mathbb{N}^{r_{d_{1}}}$, $|r|=r_{1}+\cdots+r_{d_{1}}$. Moreover $\langle\cdot, \cdot\rangle$ will also denote the duality pairing $\mathfrak{z}^{*} \times \mathfrak{z} \rightarrow \mathbb{R}$.

Proposition 4. Let $H: \mathbb{R}^{d_{1}} \times \mathfrak{z}^{*} \rightarrow \mathbb{C}$ be in the Schwartz class, and set

$$
\begin{aligned}
m(n, \mu, \eta)=H\left(\left(2 n_{1}+r_{1}\right) b_{1}^{\eta}+\right. & \mu_{1}, \ldots,\left(2 n_{\tilde{d}_{1}}+r_{\tilde{d}_{1}}\right) b_{\tilde{d}_{1}}^{\eta}+\mu_{\tilde{d}_{1}}, \\
& \left.\left(2 n_{\tilde{d}_{1}+1}+r_{\tilde{d}_{1}+1}\right) b_{\tilde{d}_{1}+1}^{\eta}, \ldots,\left(2 n_{d_{1}}+r_{d_{1}}\right) b_{d_{1}}^{\eta}, \eta\right)
\end{aligned}
$$

for all $n \in \mathbb{N}^{d_{1}}, \mu \in \mathbb{R}^{\tilde{d}_{1}}, \eta \in \dot{\mathfrak{z}}$. Then, for all $(z, u) \in G$,

(8) $\mathcal{K}_{H(\mathbf{L}, \mathbf{U})}(z, u)=\frac{2^{|r|}}{(2 \pi)^{\operatorname{dim} G}} \int_{\dot{\mathfrak{z}}} \int_{\mathfrak{v}} \sum_{n \in \mathbb{N}^{d_{1}}} m\left(n,\left(\left|\bar{P}_{1}^{\eta} \xi\right|^{2}, \ldots,\left|\bar{P}_{\tilde{d}_{1}}^{\eta} \xi\right|^{2}\right), \eta\right)$

$$
\times\left[\prod_{j=1}^{d_{1}} \mathcal{L}_{n_{j}}^{\left(r_{j}-1\right)}\left(\left|P_{j}^{\eta} \xi\right|^{2} / b_{j}^{\eta}\right)\right] e^{i\langle\xi, z\rangle} e^{i\langle\eta, u\rangle} d \xi d \eta
$$

Proof. For all $\eta \in \dot{\mathfrak{z}}$ and $j \in\left\{1, \ldots, d_{1}\right\}$, let $E_{j, 1}^{\eta}, \bar{E}_{j, 1}^{\eta}, \ldots, E_{j, r_{j}}^{\eta}, \bar{E}_{j, r_{j}}^{\eta}$ be an orthonormal basis of the range of $P_{j}^{\eta}$ such that

$$
J_{\eta} E_{j, l}^{\eta}=b_{j}^{\eta} \bar{E}_{j, l}^{\eta}, \quad J_{\eta} \bar{E}_{j, l}^{\eta}=-b_{j}^{\eta} E_{j, l}^{\eta}, \quad \text { for } l=1, \ldots, r_{j} .
$$

Hence, for all $z \in \mathfrak{v}, \eta \in \dot{\mathfrak{z}}$, and $j \in\left\{1, \ldots, d_{1}\right\}$, we can write

$$
P_{j}^{\eta} z=\sum_{l=1}^{r_{j}}\left(z_{j, l}^{\eta} E_{j}^{\eta}+\bar{z}_{j, l}^{\eta} \bar{E}_{j, l}^{\eta}\right)
$$

for some uniquely determined $z_{j, l}^{\eta}, \bar{z}_{j, l}^{\eta} \in \mathbb{R}$; set then $z_{j}^{\eta}=\left(z_{j, 1}^{\eta}, \ldots, z_{j, r_{j}}^{\eta}\right), \bar{z}_{j}^{\eta}=$ $\left(\bar{z}_{j, 1}^{\eta}, \ldots, \bar{z}_{j, r_{j}}^{\eta}\right)$, and moreover $z^{\eta}=\left(z_{1}^{\eta}, \ldots, z_{d_{1}}^{\eta}\right)$ and $\bar{z}^{\eta}=\left(\bar{z}_{1}^{\eta}, \ldots, \bar{z}_{d_{1}}^{\eta}\right)$.

For all $\eta \in \dot{z}$ and all $\rho \in \operatorname{ker} J_{\eta}$, an irreducible unitary representation $\pi_{\eta, \rho}$ of $G$ on $L^{2}\left(\mathbb{R}^{r}\right)$ is defined by

$$
\pi_{\eta, \rho}(z, u) \phi(v)=e^{i\langle\eta, u\rangle} e^{i\left\langle\rho, \bar{P}^{\eta} z\right\rangle} e^{i \sum_{j=1}^{d_{1}} b_{j}^{\eta}\left\langle v+z_{j}^{\eta} / 2, \bar{z}_{j}^{\eta}\right\rangle} \phi\left(z^{\eta}+v\right)
$$

for all $(z, u) \in G, v \in \mathbb{R}^{r}, \phi \in L^{2}\left(\mathbb{R}^{r}\right)$, where $\bar{P}^{\eta}=\bar{P}_{1}^{\eta}+\cdots+\bar{P}_{\tilde{d}_{1}}^{\eta}$ is the orthogonal projection onto ker $J_{\eta}$. Following, e.g., [1, §2], one can see that these representations are sufficient to write the Plancherel formula for the group Fourier transform of $G$, and the corresponding Fourier inversion formula:

$$
f(z, u)=(2 \pi)^{|r|-\operatorname{dim} G} \int_{\dot{\mathfrak{z}}} \int_{\operatorname{ker} J_{\eta}} \operatorname{tr}\left(\pi_{\eta, \rho}(z, u) \pi_{\eta, \rho}(f)\right) \prod_{j=1}^{d_{1}}\left(b_{j}^{\eta}\right)^{r_{j}} d \rho d \eta
$$

for all $f: G \rightarrow \mathbb{C}$ in the Schwartz class and all $(z, u) \in G$, where $\pi_{\eta, \rho}(f)=$ $\int_{G} f(g) \pi_{\eta, \rho}\left(g^{-1}\right) d g$.

Fix $\eta \in \dot{\mathfrak{z}}$ and $\rho \in \operatorname{ker} J_{\eta}$. The operators (6) are represented in $\pi_{\eta, \rho}$ as

$$
d \pi_{\eta, \rho}\left(L_{j}\right)=-\Delta_{v_{j}}^{2}+\left(b_{j}^{\eta}\right)^{2}\left|v_{j}\right|^{2}+\left|P_{j} \rho\right|^{2}, \quad d \pi_{\eta, \rho}\left(-i U_{k}\right)=\eta_{k},
$$

for all $j \in\left\{1, \ldots, d_{1}\right\}$ and $k \in\left\{1, \ldots, d_{2}\right\}$, where $v_{j} \in \mathbb{R}^{r_{j}}$ denotes the $j$-th component of $v \in \mathbb{R}^{r}$, and $\Delta_{v_{j}}$ denotes the corresponding partial Laplacian. Let $h_{\ell}$ denote the $\ell$-th Hermite function, that is,

$$
h_{\ell}(t)=(-1)^{\ell}\left(\ell ! 2^{\ell} \sqrt{\pi}\right)^{-1 / 2} e^{t^{2} / 2}\left(\frac{d}{d t}\right)^{\ell} e^{-t^{2}},
$$


and, for all $\omega \in \mathbb{N}^{r}$, define $\tilde{h}_{\eta, \omega}: \mathbb{R}^{r} \rightarrow \mathbb{R}$ by

$$
\tilde{h}_{\eta, \omega}=\tilde{h}_{\eta, \omega, 1} \otimes \cdots \otimes \tilde{h}_{\eta, \omega, d_{1}}, \quad \tilde{h}_{\eta, \omega, j}\left(v_{j}\right)=\left(b_{j}^{\eta}\right)^{r_{j} / 4} \prod_{l=1}^{r_{j}} h_{\omega_{j, l}}\left(\left(b_{j}^{\eta}\right)^{1 / 2} v_{j, l}\right),
$$

for all $j \in\left\{1, \ldots, d_{1}\right\}$, where $\omega_{j, l}$ and $v_{j, l}$ denote the $l$-th components of $\omega_{j} \in \mathbb{N}^{r_{j}}$ and $v_{j} \in \mathbb{R}^{r_{j}}$. Then $\left\{\tilde{h}_{\eta, \omega}\right\}_{\omega \in \mathbb{N}^{r}}$ is a complete orthonormal system for $L^{2}\left(\mathbb{R}^{r}\right)$, made of joint eigenfunctions of the operators (10). In fact,

$$
\begin{aligned}
d \pi_{\eta, \rho}\left(L_{j}\right) \tilde{h}_{\eta, \omega} & =\left(\left(2\left|\omega_{j}\right|+r_{j}\right) b_{j}^{\eta}+\left|P_{j} \rho\right|^{2}\right) \tilde{h}_{\eta, \omega}, \\
d \pi_{\eta, \rho}\left(-i U_{k}\right) \tilde{h}_{\eta, \omega} & =\eta_{k} \tilde{h}_{\eta, \omega},
\end{aligned}
$$

where $\left|\omega_{j}\right|=\omega_{j, 1}+\cdots+\omega_{j, r_{j}}$; it should be observed that $P_{j} \rho=0$ if $j>\tilde{d}_{1}$.

Since $H: \mathbb{R}^{d_{1}} \times \mathfrak{z}^{*} \rightarrow \mathbb{C}$ is in the Schwartz class, $\mathcal{K}_{H(\mathbf{L}, \mathbf{U})}: G \rightarrow \mathbb{C}$ is in the Schwartz class too (see [2, Theorem 5.2] or [10, §4.2]). Moreover

$$
\pi_{\eta, \rho}\left(\mathcal{K}_{H(\mathbf{L}, \mathbf{U})}\right) \tilde{h}_{\eta, \omega}=m\left(\left(\left|\omega_{1}\right|, \ldots,\left|\omega_{d_{1}}\right|\right),\left(\left|P_{1} \rho\right|^{2}, \ldots,\left|P_{\tilde{d}_{1}} \rho\right|^{2}\right), \eta\right) \tilde{h}_{\eta, \omega}
$$

by (11) and [18, Proposition 1.1]; hence, if $\varphi_{\eta, \rho, \omega}(z, u)=\left\langle\pi_{\eta, \rho}(z, u) \tilde{h}_{\eta, \omega}, \tilde{h}_{\eta, \omega}\right\rangle$ is the corresponding diagonal matrix coefficient of $\pi_{\eta, \rho}$, then

$$
\left\langle\pi_{\eta, \rho}(z, u) \pi_{\eta, \rho}\left(\mathcal{K}_{H(\mathbf{L}, \mathbf{U})}\right) \tilde{h}_{\eta, \omega}, \tilde{h}_{\eta, \omega}\right\rangle=m\left(\left(\left|\omega_{j}\right|\right)_{j \leq d_{1}},\left(\left|P_{j} \rho\right|^{2}\right)_{j \leq \tilde{d}_{1}}, \eta\right) \varphi_{\eta, \rho, \omega}(z, u) .
$$

Therefore (9) gives that

$$
\begin{aligned}
& \mathcal{K}_{H(\mathbf{L}, \mathbf{U})}(z, u) \\
= & (2 \pi)^{|r|-\operatorname{dim} G} \int_{\dot{\mathfrak{z}}} \int_{\operatorname{ker} J_{\eta}} \sum_{n \in \mathbb{N}^{d_{1}}} m\left(n,\left(\left|P_{j} \rho\right|^{2}\right)_{j \leq \tilde{d}_{1}}, \eta\right) \psi_{\eta, \rho, n}(z, u) \prod_{j=1}^{d_{1}}\left(b_{j}^{\eta}\right)^{r_{j}} d \rho d \eta,
\end{aligned}
$$

where

$$
\psi_{\eta, \rho, n}(z, u)=\sum_{\substack{\omega \in \mathbb{N}^{r} \\\left|\omega_{1}\right|=n_{1}, \ldots,\left|\omega_{d_{1}}\right|=n_{d_{1}}}} \varphi_{\eta, \rho, \omega}(z, u) .
$$

On the other hand,

$$
\begin{aligned}
\varphi_{\eta, \rho, \omega}(z, u) & =e^{i\langle\eta, u\rangle} e^{i\left\langle\rho, \bar{P}^{\eta} z\right\rangle} \prod_{j=1}^{d_{1}} \prod_{l=1}^{r_{j}}\left[\left(b_{j}^{\eta}\right)^{1 / 2}\right. \\
& \left.\times \int_{\mathbb{R}} e^{i b_{j}^{\eta} s \bar{z}_{j, l}^{\eta}} h_{\omega_{j, l}}\left(\left(b_{j}^{\eta}\right)^{1 / 2}\left(s+z_{j, l}^{\eta} / 2\right)\right) h_{\omega_{j, l}}\left(\left(b_{j}^{\eta}\right)^{1 / 2}\left(s-z_{j, l}^{\eta} / 2\right)\right) d s\right] .
\end{aligned}
$$

The last integral is essentially the Fourier-Wigner transform of a pair of Hermite functions, whose bidimensional Fourier transform is a Fourier-Wigner transform too [7, formula (1.90)]. The parity properties of the Hermite functions then yield

$$
\begin{aligned}
& \varphi_{\eta, \rho, \omega}(z, u)=e^{i\langle\eta, u\rangle} e^{i\left\langle\rho, \bar{P}^{\eta} z\right\rangle} \prod_{j=1}^{d_{1}} \prod_{l=1}^{j}\left[\frac{(-1)^{\omega_{j, l}}}{\pi b_{j}^{\eta}} \int_{\mathbb{R} \times \mathbb{R}} e^{i \theta_{1} z_{j, l}^{\eta}} e^{i \theta_{2} \bar{z}_{j, l}^{\eta}}\right. \\
&\left.\times \int_{\mathbb{R}} e^{i t\left(2 \theta_{1} /\left(b_{j}^{\eta}\right)^{1 / 2}\right)} h_{\omega_{j, l}}\left(t+\theta_{2} /\left(b_{j}^{\eta}\right)^{1 / 2}\right) h_{\omega_{j, l}}\left(t-\theta_{2} /\left(b_{j}^{\eta}\right)^{1 / 2}\right) d t d \theta_{1} d \theta_{2}\right] .
\end{aligned}
$$

Since the Fourier-Wigner transform of a pair of Hermite functions can be expressed in terms of Laguerre polynomials (see [7, Theorem 1.104] or [21, Theorem 1.3.4]), 
we obtain that

$$
\begin{aligned}
\varphi_{\eta, \rho, \omega}(z, u)=\frac{e^{i\langle\eta, u\rangle} e^{i\left\langle\rho, \bar{P}^{\eta} z\right\rangle}}{\pi^{|r|}} & \int_{\mathbb{R}^{r} \times \mathbb{R}^{r}} e^{i\left\langle\zeta_{1}, z^{\eta}\right\rangle} e^{i\left\langle\zeta_{2}, \bar{z}^{\eta}\right\rangle} \\
& \times \prod_{j=1}^{d_{1}}\left[\left(b_{j}^{\eta}\right)^{-r_{j}} \prod_{l=1}^{r_{j}} \mathcal{L}_{\omega_{j, l}}^{(0)}\left(\left(\zeta_{1, j, l}^{2}+\zeta_{2, j, l}^{2}\right) / b_{j}^{\eta}\right)\right] d \zeta_{1} d \zeta_{2}
\end{aligned}
$$

Consequently, for all $n \in \mathbb{N}^{d_{1}}$,

$$
\begin{aligned}
\psi_{\eta, \rho, n}(z, u)=\frac{e^{i\langle\eta, u\rangle} e^{i\left\langle\rho, \bar{P}^{\eta} z\right\rangle}}{\pi^{|r|}} \int_{\mathbb{R}^{r} \times \mathbb{R}^{r}} e^{i\left\langle\zeta_{1}, z^{\eta}\right\rangle} e^{i\left\langle\zeta_{2}, \bar{z}^{\eta}\right\rangle} \\
\times \prod_{j=1}^{d_{1}}\left[\left(b_{j}^{\eta}\right)^{-r_{j}} 2^{r_{j}-1} \mathcal{L}_{n_{j}}^{\left(r_{j}-1\right)}\left(\left(\left|\zeta_{1, j}\right|^{2}+\left|\zeta_{2, j}\right|^{2}\right) / b_{j}^{\eta}\right)\right] d \zeta_{1} d \zeta_{2}
\end{aligned}
$$

[5. $§ 10.12$, formula (41)]. The conclusion then follows by plugging (13) into (12) and performing a change of variable by rotation in the inner integrals.

\section{A weighted Plancherel estimate}

Proposition 4 expresses the convolution kernel $\mathcal{K}_{H(\mathbf{L}, \mathbf{U})}$ as the inverse Fourier transform of a function of the multiplier $H$. Due to the properties of the Fourier transform, it is not unreasonable to think that multiplying the kernel by a polynomial weight might correspond to taking derivatives of the multiplier. As a matter of fact, the presence of the Laguerre expansion leads us to consider both "discrete" and "continuous" derivatives of the reparametrization $m: \mathbb{N}^{d_{1}} \times \mathbb{R}^{\tilde{d}_{1}} \times \dot{\mathfrak{z}} \rightarrow \mathbb{C}$ of the multiplier $H$ given by (7).

For convenience, set $\mathcal{L}_{n}^{(k)}=0$ for all $n<0$. From the properties of Laguerre polynomials (see, e.g., [5, §10.12]) one can easily derive the following identities.

Lemma 5. For all $k, n, m \in \mathbb{N}$ and $t \in \mathbb{R}$,

$$
\begin{gathered}
\mathcal{L}_{n}^{(k)}(t)=\mathcal{L}_{n-1}^{(k+1)}(t)+\mathcal{L}_{n}^{(k+1)}(t), \\
\frac{d}{d t} \mathcal{L}_{n}^{(k)}(t)=\mathcal{L}_{n-1}^{(k+1)}(t)-\mathcal{L}_{n}^{(k+1)}(t), \\
\int_{0}^{\infty} \mathcal{L}_{n}^{(k)}(t) \mathcal{L}_{m}^{(k)}(t) t^{k} d t= \begin{cases}\frac{(n+k) !}{2^{k+1} n !} & \text { if } n=m \\
0 & \text { otherwise }\end{cases}
\end{gathered}
$$

Let $e_{1}, \ldots, e_{d_{1}}$ denote the standard basis of $\mathbb{R}^{d_{1}}$. We introduce some operators on functions $f: \mathbb{N}^{d_{1}} \times \mathbb{R}^{\tilde{d_{1}}} \times \dot{\mathfrak{z}} \rightarrow \mathbb{C}$ :

$$
\begin{aligned}
\tau_{j} f(n, \mu, \eta) & =f\left(n+e_{j}, \mu, \eta\right), \\
\delta_{j} f(n, \mu, \eta) & =f\left(n+e_{j}, \mu, \eta\right)-f(n, \mu, \eta), \\
\partial_{\mu_{l}} f(n, \mu, \eta) & =\frac{\partial}{\partial \mu_{l}} f(n, \mu, \eta), \\
\partial_{\eta_{k}} f(n, \mu, \eta) & =\frac{\partial}{\partial \eta_{k}} f(n, \mu, \eta)
\end{aligned}
$$

for all $j \in\left\{1, \ldots, d_{1}\right\}, l \in\left\{1, \ldots, \tilde{d}_{1}\right\}, k \in\left\{1, \ldots, d_{2}\right\}$.

For all $h \in \mathbb{N}$ and all multiindices $\alpha \in \mathbb{N}^{h}$, we denote by $|\alpha|$ the length $\alpha_{1}+\cdots+$ $\alpha_{h}$ of $\alpha$. Inequalities between multiindices, such as $\alpha \leq \alpha^{\prime}$, shall be interpreted componentwise. Set moreover $(\alpha)_{+}=\left(\left(\alpha_{1}\right)_{+}, \ldots,\left(\alpha_{h}\right)_{+}\right)$, where $(\ell)_{+}=\max \{\ell, 0\}$. 
A function $\Psi: \dot{\mathfrak{z}} \times \mathfrak{v} \rightarrow \mathbb{C}$ will be called multihomogeneous if there exist $h_{0}, h_{1}, \ldots, h_{d_{1}} \in \mathbb{R}$ such that

$$
\Psi\left(\lambda_{0} \eta, \sum_{j=1}^{d_{1}} \lambda_{j} P_{j} \xi\right)=\lambda_{0}^{h_{0}} \lambda_{1}^{h_{1}} \ldots \lambda_{d_{1}}^{h_{d_{1}}} \Psi(\eta, \xi)
$$

for all $\left.\eta \in \dot{\mathfrak{z}}, \xi \in \mathfrak{v}, \lambda_{0}, \lambda_{1}, \ldots, \lambda_{d_{1}} \in\right] 0, \infty\left[\right.$; the degrees of homogeneity $h_{0}, h_{1}, \ldots, h_{d_{1}}$ of $\Psi$ will also be denoted as $\operatorname{deg}_{\mathfrak{z}} \Psi, \operatorname{deg}_{\mathfrak{v}_{1}} \Psi, \ldots, \operatorname{deg}_{\mathfrak{v}_{d_{1}}} \Psi$. Note that, if $\Psi$ is multihomogeneous and continuous, then $\operatorname{deg}_{\mathfrak{v}_{j}} \Psi \geq 0$ for all $j \in\left\{1, \ldots, d_{1}\right\}$.

Proposition 6. Let $H: \mathbb{R}^{d_{1}} \times \mathfrak{z}^{*} \rightarrow \mathbb{C}$ be smooth and compactly supported in $\mathbb{R}^{d_{1}} \times \dot{\mathfrak{z}}$, and let $m(n, \mu, \eta)$ be defined by (7). For all $\alpha \in \mathbb{N}^{d_{2}}$,

$$
\begin{aligned}
u^{\alpha} \mathcal{K}_{H(\mathbf{L}, \mathbf{U})}(z, u)= & \sum_{\iota \in I_{\alpha}} \int_{\dot{\mathfrak{z}}} \int_{\mathfrak{v}} \sum_{n \in \mathbb{N}^{d_{1}}} \partial_{\eta}^{\gamma^{\iota}} \partial_{\mu}^{\theta^{\iota}} \delta^{\beta^{\iota}} m\left(n,\left(\left|\bar{P}_{j}^{\eta} \xi\right|^{2}\right)_{j \leq \tilde{d_{1}}}, \eta\right) \\
& \times \Psi_{\iota}(\eta, \xi)\left[\prod_{j=1}^{d_{1}} \mathcal{L}_{n_{j}}^{\left(r_{j}-1+\beta_{j}^{\iota}\right)}\left(\left|P_{j}^{\eta} \xi\right|^{2} / b_{j}^{\eta}\right)\right] e^{i\langle\xi, z\rangle} e^{i\langle\eta, u\rangle} d \xi d \eta,
\end{aligned}
$$

for almost all $(z, u) \in G$, where $I_{\alpha}$ is a finite set and, for all $\iota \in I_{\alpha}$,

- $\gamma^{\iota} \in \mathbb{N}^{d_{2}}, \theta^{\iota} \in \mathbb{N}^{\tilde{d}_{1}}, \beta^{\iota} \in \mathbb{N}^{d_{1}}, \gamma^{\iota} \leq \alpha$,

- $\Psi_{\iota}=\Psi_{\iota, 0} \Psi_{\iota, 1} \ldots \Psi_{\iota, d_{1}}$, where $\Psi_{\iota, j}: \dot{\mathfrak{z}} \times \mathfrak{v} \rightarrow \mathbb{C}$ is smooth and multihomogeneous for all $j \in\left\{0, \ldots, d_{1}\right\}$,

- $\operatorname{deg}_{\mathfrak{z}} \Psi_{\iota}=\left|\gamma^{\iota}\right|-|\alpha|-\left|\beta^{\iota}\right|$ and $\operatorname{deg}_{\mathfrak{v}_{j}} \Psi_{\iota}=2 \beta_{j}^{\iota}+2 \theta_{j}^{\iota}$ for all $j \in\left\{1, \ldots, d_{1}\right\}$,

- for all $j \in\left\{1, \ldots, d_{1}\right\}, \Psi_{\iota, j}(\eta, \xi)$ is a product of factors of the form $\left|P_{j}^{\eta} \xi\right|^{2}$ or $\partial_{\eta_{k}}\left|P_{j}^{\eta} \xi\right|^{2}$ for $k \in\left\{1, \ldots, d_{2}\right\}$,

- $\left|\gamma^{\iota}\right|+\left|\theta^{\iota}\right|+\left|\beta^{\iota}\right|+\sum_{j=1}^{d_{1}}\left(\beta_{j}^{\iota}-\left(\operatorname{deg}_{\mathfrak{v}_{j}} \Psi_{\iota, j}\right) / 2\right)_{+} \leq|\alpha|$.

Proof. By Proposition 4 and the properties of the Fourier transform, we are reduced to proving that, for all $\alpha \in \mathbb{N}^{d_{2}}, \eta \in \dot{\mathfrak{z}}, \xi \in \mathfrak{v}$,

$$
\begin{aligned}
& \left(\frac{\partial}{\partial \eta}\right)^{\alpha} \sum_{n \in \mathbb{N}^{d_{1}}} m\left(n,\left(\left|\bar{P}_{j}^{\eta} \xi\right|^{2}\right)_{j \leq \tilde{d}_{1}}, \eta\right) \prod_{j=1}^{d_{1}} \mathcal{L}_{n_{j}}^{\left(r_{j}-1\right)}\left(\left|P_{j}^{\eta} \xi\right|^{2} / b_{j}^{\eta}\right) \\
& =\sum_{\iota \in I_{\alpha}} \sum_{n \in \mathbb{N}^{d_{1}}} \partial_{\eta}^{\gamma^{\iota}} \partial_{\mu}^{\theta^{\iota}} \delta^{\beta^{\iota}} m\left(n,\left(\left|\bar{P}_{j}^{\eta} \xi\right|^{2}\right)_{j \leq \tilde{d_{1}}}, \eta\right) \Psi_{\iota}(\eta, \xi) \prod_{j=1}^{d_{1}} \mathcal{L}_{n_{j}}^{\left(r_{j}-1+\beta_{j}^{\iota}\right)}\left(\left|P_{j}^{\eta} \xi\right|^{2} / b_{j}^{\eta}\right),
\end{aligned}
$$

where $I_{\alpha}, \gamma^{\iota}, \theta^{\iota}, \beta^{\iota}, \Psi_{\iota}$ are as in the above statement.

This is easily proved by induction on $|\alpha|$. For $|\alpha|=0$ it is trivially verified. For the inductive step, one applies Leibniz' rule, and exploits the following observations:

- when a derivative $\partial_{\eta_{k}}$ hits a Laguerre function, by the identity (15) and summation by parts, the type of the Laguerre function is increased by 1 , as well as the corresponding component of $\beta^{\iota}$;

- for all $j \in\left\{1, \ldots, d_{1}\right\}, b_{j}^{\eta}=\sqrt{\operatorname{tr}\left(-J_{\eta}^{2} P_{j}\right) /\left(2 r_{j}\right)}$ is a smooth function of $\eta \in \dot{\mathfrak{z}}$, homogeneous of degree 1 ;

- for all $j \in\left\{1, \ldots, d_{1}\right\}, P_{j}^{\eta}=-J_{\eta}^{2} P_{j} /\left(b_{j}^{\eta}\right)^{2}$ is a smooth function of $\eta \in \dot{\mathfrak{z}}$, homogeneous of degree 0 , and in fact it is constant if $j>\tilde{d}_{1}$;

- for all $j \in\left\{1, \ldots, \tilde{d}_{1}\right\},\left|P_{j}^{\eta} \xi\right|^{2}=\left\langle P_{j}^{\eta} P_{j} \xi, P_{j} \xi\right\rangle$ is a smooth bihomogeneous function of $\left(\eta, P_{j} \xi\right) \in \dot{\mathfrak{z}} \times \mathfrak{v}_{j}$ of bidegree $(0,2)$, and moreover

$$
\begin{gathered}
\left|\bar{P}_{j}^{\eta} \xi\right|^{2}=\left|P_{j} \xi\right|^{2}-\left|P_{j}^{\eta} \xi\right|^{2}, \quad \partial_{\eta_{k}}\left|\bar{P}_{j}^{\eta} \xi\right|^{2}=-\partial_{\eta_{k}}\left|P_{j}^{\eta} \xi\right|^{2}, \\
\partial_{\eta_{k}}\left(\left|P_{j}^{\eta} \xi\right|^{2} / b_{j}^{\eta}\right)=\left|P_{j}^{\eta} \xi\right|^{2} \partial_{\eta_{k}}\left(1 / b_{j}^{\eta}\right)+\left(\partial_{\eta_{k}}\left|P_{j}^{\eta} \xi\right|^{2}\right) / b_{j}^{\eta}
\end{gathered}
$$

for all $k \in\left\{1, \ldots, d_{2}\right\}$. 
The conclusion follows.

Note that, for all $j \in\left\{1, \ldots, d_{1}\right\}, \mu \in \mathbb{R}^{\tilde{d}_{1}}, \eta \in \dot{\mathfrak{z}}$, the quantities $\tau_{j} f(\cdot, \mu, \eta)$, $\delta_{j} f(\cdot, \mu, \eta)$ depend only on $f(\cdot, \mu, \eta)$; in other words, $\tau_{j}$ and $\delta_{j}$ can be considered as operators on functions $\mathbb{N}^{d_{1}} \rightarrow \mathbb{C}$.

The following lemma exploits the orthogonality properties (16) of the Laguerre functions, together with (14), and shows that a mismatch between the type of the Laguerre function and the exponent of the weight attached to the measure may be turned in some cases into discrete differentiation.

Lemma 7. For all $h, k \in \mathbb{N}^{d_{1}}$ and all compactly supported $f: \mathbb{N}^{d_{1}} \rightarrow \mathbb{C}$,

$$
\begin{aligned}
\int_{] 0, \infty\left[d_{1}\right.}\left|\sum_{n \in \mathbb{N}^{d_{1}}} f(n) \prod_{j=1}^{d_{1}} \mathcal{L}_{n_{j}}^{\left(k_{j}\right)}\left(t_{j}\right)\right|^{2} t^{h} d t & \\
& \leq C_{h, k} \sum_{n \in \mathbb{N}^{d_{1}}}\left|\delta^{(k-h)_{+}} f(n)\right|^{2} \prod_{j=1}^{d_{1}}\left(1+n_{j}\right)^{h_{j}+2\left(k_{j}-h_{j}\right)_{+}} .
\end{aligned}
$$

Proof. Via an inductive argument, we may reduce to the case $d_{1}=1$.

Note that, if $f$ is compactly supported, then $\tau^{l} f$ is null for all sufficiently large $l \in \mathbb{N}$. Hence the operator $1+\tau$, when restricted to the set of compactly supported functions, is invertible, with inverse given by

$$
(1+\tau)^{-1} f=\sum_{l \in \mathbb{N}}(-1)^{l} \tau^{l} f .
$$

Then by (14) we deduce that, for all $k \in \mathbb{N}$,

$$
\begin{aligned}
\sum_{n \in \mathbb{N}} f(n) \mathcal{L}_{n}^{(k)}(t) & =\sum_{n \in \mathbb{N}}(1+\tau) f(n) \mathcal{L}_{n}^{(k+1)}(t), \\
\sum_{n \in \mathbb{N}} f(n) \mathcal{L}_{n}^{(k+1)}(t) & =\sum_{n \in \mathbb{N}}(1+\tau)^{-1} f(n) \mathcal{L}_{n}^{(k)}(t),
\end{aligned}
$$

and consequently, for all $h, k \in \mathbb{N}$,

$$
\sum_{n \in \mathbb{N}} f(n) \mathcal{L}_{n}^{(k)}(t)=\sum_{n \in \mathbb{N}}(1+\tau)^{h-k} f(n) \mathcal{L}_{n}^{(h)}(t)
$$

Thus the orthogonality properties (16) of the Laguerre functions give us that

$$
\int_{0}^{\infty}\left|\sum_{n \in \mathbb{N}} f(n) \mathcal{L}_{n}^{(k)}(t)\right|^{2} t^{h} d t \leq C_{h, k} \sum_{n \in \mathbb{N}}\left|(1+\tau)^{h-k} f(n)\right|^{2}\langle n\rangle^{h},
$$

where $\langle n\rangle=1+n$.

In the case $h \geq k,(1+\tau)^{h-k}$ is given by the finite sum

$$
(1+\tau)^{h-k}=\sum_{\ell=0}^{h-k}\left(\begin{array}{c}
h-k \\
\ell
\end{array}\right) \tau^{\ell}
$$

and the conclusion follows immediately by the triangular inequality.

In the case $h<k$, instead, since $\delta=\tau-1$, from the identity $1-\tau^{2}=(1-\tau)(1+\tau)$ we deduce that

$$
(1+\tau)^{h-k}=(-\delta)^{k-h}\left(1-\tau^{2}\right)^{h-k}=(-1)^{k-h} \sum_{\ell \geq 0}\left(\begin{array}{c}
\ell+k-h-1 \\
\ell
\end{array}\right) \delta^{k-h} \tau^{2 \ell},
$$


hence

$$
\begin{aligned}
\sum_{n \in \mathbb{N}}\left|(1+\tau)^{h-k} f(n)\right|^{2}\langle n\rangle^{h} & =\sum_{n \in \mathbb{N}}\left|\sum_{\ell \geq 0}\left(\begin{array}{c}
\ell+k-h-1 \\
\ell
\end{array}\right) \delta^{k-h} f(n+2 \ell)\right|^{2}\langle n\rangle^{h} \\
& \leq C_{h, k} \sum_{n \in \mathbb{N}}\left|\sum_{\ell \geq n}\langle\ell\rangle^{k-h-1} \delta^{k-h} f(\ell)\right|^{2}\langle n\rangle^{h} \\
& \leq C_{h, k} \sum_{n \in \mathbb{N}}\langle n\rangle^{-1 / 2} \sum_{\ell \geq n}\left|\langle\ell\rangle^{k-h-1 / 4} \delta^{k-h} f(\ell)\right|^{2}\langle n\rangle^{h} \\
& \leq C_{h, k} \sum_{\ell \in \mathbb{N}}\langle\ell\rangle^{2 k-2 h-1 / 2}\left|\delta^{k-h} f(\ell)\right|^{2} \sum_{n=0}^{\ell}\langle n\rangle^{h-1 / 2} \\
& \leq C_{h, k} \sum_{\ell \in \mathbb{N}}\langle\ell\rangle^{2 k-h}\left|\delta^{k-h} f(\ell)\right|^{2},
\end{aligned}
$$

by the Cauchy-Schwarz inequality, and we are done.

Let $|\cdot|$ denote any Euclidean norm on $\mathfrak{z}^{*}$. The previous lemma, together with Plancherel's formula for the Fourier transform, yields the following $L^{2}$ estimate.

Proposition 8. Under the hypotheses of Proposition [6, for all $\alpha \in \mathbb{N}^{d_{2}}$,

$$
\begin{aligned}
\int_{G} \mid u^{\alpha} \mathcal{K}_{H(\mathbf{L}, \mathbf{U})} & \left.(z, u)\right|^{2} d z d u \leq C_{\alpha} \sum_{\iota \in \tilde{I}_{\alpha}} \int_{\dot{\mathfrak{z}}} \int_{\left[0, \infty\left[{ }^{\tilde{d_{1}}}\right.\right.} \sum_{n \in \mathbb{N}^{d_{1}}}\left|\partial_{\eta}^{\gamma^{\iota}} \partial_{\mu}^{\theta^{\iota}} \delta^{\beta^{\iota}} m(n, \mu, \eta)\right|^{2} \\
& \times|\eta|^{2\left|\gamma^{\iota}\right|-2|\alpha|-2\left|\beta^{\iota}\right|+\left|a^{\iota}\right|+d_{1}}\left(1+n_{1}\right)^{a_{1}^{\iota}} \ldots\left(1+n_{d_{1}}\right)^{a_{d_{1}}^{\iota}} d \sigma_{\iota}(\mu) d \eta,
\end{aligned}
$$

where $\tilde{I}_{\alpha}$ is a finite set and, for all $\iota \in \tilde{I}_{\alpha}$,

- $\gamma^{\iota} \in \mathbb{N}^{d_{2}}, \theta^{\iota} \in \mathbb{N}^{\tilde{d}_{1}}, a^{\iota}, \beta^{\iota} \in \mathbb{N}^{d_{1}}$,

- $\gamma^{\iota} \leq \alpha,\left|\gamma^{\iota}\right|+\left|\theta^{\iota}\right|+\left|\beta^{\iota}\right| \leq|\alpha|$,

- $\sigma_{\iota}$ is a regular Borel measure on $\left[0, \infty\left[{ }^{\tilde{d_{1}}}\right.\right.$.

Proof. Note that, for all $j \in\left\{1, \ldots, d_{1}\right\}$,

$$
\partial_{\eta_{k}}\left(\left|P_{j}^{\eta} \xi\right|^{2}\right)=2\left\langle\left(\partial_{\eta_{k}} P_{j}^{\eta}\right) P_{j} \xi, P_{j}^{\eta} \xi\right\rangle \leq C|\eta|^{-1}\left|P_{j}^{\eta} \xi\right|\left|P_{j} \xi\right|
$$

consequently, if $\Psi_{\iota}, \Psi_{\iota, j}, \gamma^{\iota}, \theta^{\iota}, \beta^{\iota}$ are as in the statement of Proposition 6, then

$$
\left|\Psi_{\iota, j}(\eta, \xi)\right|^{2} \leq C_{\iota}|\eta|^{2 \operatorname{deg}_{\mathfrak{z}} \Psi_{\iota, j}}\left|P_{j}^{\eta} \xi\right|^{\operatorname{deg}_{\mathfrak{v}_{j}} \Psi_{\iota, j}}\left|P_{j} \xi\right|^{\operatorname{deg}_{\mathfrak{v}_{j}} \Psi_{\iota, j}}
$$

for all $j \in\left\{1, \ldots, d_{1}\right\}$, hence

$$
\begin{aligned}
\left|\Psi_{\iota}(\eta, \xi)\right|^{2} & \leq C_{\iota}|\eta|^{2 \operatorname{deg}_{\mathfrak{z}} \Psi_{\iota}} \prod_{j=1}^{d_{1}}\left|P_{j}^{\eta} \xi\right|^{\operatorname{deg}_{\mathfrak{v}_{j}} \Psi_{\iota, j}}\left|P_{j} \xi\right|^{2 \operatorname{deg}_{\mathfrak{v}_{j}} \Psi_{\iota, 0}+\operatorname{deg}_{\mathfrak{v}_{j}} \Psi_{\iota, j}} \\
& \leq C_{\iota}|\eta|^{2\left|\gamma^{\iota}\right|-2|\alpha|-2\left|\beta^{\iota}\right|} \prod_{j=1}^{d_{1}} \sum_{h_{j}=\left(\operatorname{deg}_{\mathfrak{v}_{j}} \Psi_{\iota, j}\right) / 2}^{2 \theta_{j}^{\iota}+2 \beta_{j}^{\iota}}\left|P_{j}^{\eta} \xi\right|^{2 h_{j}}\left|\bar{P}_{j}^{\eta} \xi\right|^{4 \theta_{j}^{\iota}+4 \beta_{j}^{\iota}-2 h_{j}},
\end{aligned}
$$

and moreover, for all $h \in \mathbb{N}^{d_{1}}$, if $h_{j} \geq\left(\operatorname{deg}_{\mathfrak{v}_{j}} \Psi_{\iota, j}\right) / 2$ for all $j \in\left\{1, \ldots, d_{1}\right\}$, then

$$
\left|\gamma^{\iota}\right|+\left|\theta^{\iota}\right|+\left|\beta^{\iota}\right|+\sum_{j=1}^{\tilde{d}_{1}}\left(\beta_{j}^{\iota}-h_{j}\right)_{+} \leq|\alpha| .
$$


By Proposition 6. Plancherel's formula and the triangular inequality, we then obtain that the left-hand side of (17) is majorized by a finite sum of terms of the form

$$
\begin{aligned}
\left.\int_{\dot{\mathfrak{z}}} \int_{\mathfrak{v}} \sum_{n \in \mathbb{N}^{d_{1}}} \partial_{\eta}^{\gamma} \partial_{\mu}^{\theta} \delta^{\beta} m\left(n,\left(\left|\bar{P}_{j}^{\eta} \xi\right|^{2}\right)_{j \leq \tilde{d_{1}}}, \eta\right) \prod_{j=1}^{d_{1}} \mathcal{L}_{n_{j}}^{\left(r_{j}-1+\beta_{j}\right)}\left(\left|P_{j}^{\eta} \xi\right|^{2} / b_{j}^{\eta}\right)\right|^{2} \\
\times|\eta|^{2|\gamma|-2|\alpha|-2|\beta|} \prod_{j=1}^{d_{1}}\left|P_{j}^{\eta} \xi\right|^{2 h_{j}} \prod_{j=1}^{\tilde{d_{1}}}\left|\bar{P}_{j}^{\eta} \xi\right|^{2 k_{j}} d \xi d \eta,
\end{aligned}
$$

where $\gamma \in \mathbb{N}^{d_{2}}, \theta, k \in \mathbb{N}^{\tilde{d}_{1}}, \beta, h \in \mathbb{N}^{d_{1}}$ and $|\gamma|+|\theta|+\left|\beta+(\beta-h)_{+}\right| \leq|\alpha|$. Simple changes of variables (rotation, polar coordinates and rescaling) allow one to rewrite (18) as a constant times

$$
\begin{aligned}
\int_{\dot{\mathfrak{z}}} \int_{] 0, \infty\left[\tilde{d}^{\tilde{1}}\right.} \int_{] 0, \infty\left[{ }^{d_{1}} \mid\right.}\left|\sum_{n \in \mathbb{N}^{d_{1}}} \partial_{\eta}^{\gamma} \partial_{\mu}^{\theta} \delta^{\beta} m(n, \mu, \eta) \prod_{j=1}^{d_{1}} \mathcal{L}_{n_{j}}^{\left(r_{j}-1+\beta_{j}\right)}\left(t_{j}\right)\right|^{2} \prod_{j=1}^{d_{1}} t_{j}^{r_{j}-1+h_{j}} d t \\
\quad \times|\eta|^{2|\gamma|-2|\alpha|-2|\beta|} \prod_{j=1}^{d_{1}}\left(b_{j}^{\eta}\right)^{h_{j}+r_{j}} \prod_{j=1}^{\tilde{d_{1}}} \mu_{j}^{k_{j}+\left(\operatorname{dim} \mathfrak{v}_{j}-2 r_{j}\right) / 2} \frac{d \mu}{\mu_{1} \cdots \mu_{\tilde{d}_{1}}} d \eta .
\end{aligned}
$$

By exploiting the fact that the $b_{j}^{\eta}$ are smooth functions of $\eta \in \dot{\mathfrak{z}}$, homogeneous of degree 1 (see the proof of Proposition [6), and applying Lemma 7 to the inner integral, the last quantity is majorized by

$$
\begin{aligned}
C \int_{\dot{\mathfrak{z}}} \int_{] 0, \infty\left[\tilde{d}^{\tilde{1}}\right.} \sum_{n \in \mathbb{N}^{d_{1}}} & \left|\partial_{\eta}^{\gamma} \partial_{\mu}^{\theta} \delta^{\beta+(\beta-h)_{+}} m(n, \mu, \eta)\right|^{2} \prod_{j=1}^{d_{1}}\left(1+n_{j}\right)^{r_{j}-1+h_{j}+2\left(\beta_{j}-h_{j}\right)_{+}} \\
& \times|\eta|^{2|\gamma|-2|\alpha|-2|\beta|+|h|+|r|} \prod_{j=1}^{\tilde{d_{1}}} \mu_{j}^{k_{j}+\left(\operatorname{dim} \mathfrak{v}_{j}-2 r_{j}\right) / 2} \frac{d \mu}{\mu_{1} \ldots \mu_{\tilde{d_{1}}}} d \eta,
\end{aligned}
$$

and since the exponents $k_{j}+\left(\operatorname{dim} \mathfrak{v}_{j}-2 r_{j}\right) / 2$ are strictly positive, while

$$
-2|\beta|+|h|+|r|=-2\left|\beta+(\beta-h)_{+}\right|+\sum_{j=1}^{d_{1}}\left(r_{j}-1+h_{j}+2\left(\beta_{j}-h_{j}\right)_{+}\right)+d_{1}
$$

and $|\gamma|+|\theta|+\left|\beta+(\beta-h)_{+}\right| \leq|\alpha|$, the conclusion follows by suitably renaming the multiindices.

\section{From discrete to CONTinuous}

Via the fundamental theorem of integral calculus, finite differences can be estimated by continuous derivatives. The next lemma is a multivariate analogue of [13. Lemma 6], and we omit the proof (see also [14, Lemma 7]).

Lemma 9. Let $f: \mathbb{N}^{d_{1}} \rightarrow \mathbb{C}$ have a smooth extension $\tilde{f}:\left[0, \infty\left[^{d_{1}} \rightarrow \mathbb{C}\right.\right.$, and let $\beta \in \mathbb{N}^{d_{1}}$. Then

$$
\delta^{\beta} f(n)=\int_{J_{\beta}} \partial^{\beta} \tilde{f}(n+s) d \nu_{\beta}(s)
$$

for all $n \in \mathbb{N}$, where $J_{\beta}=\prod_{j=1}^{d_{1}}\left[0, \beta_{j}\right]$ and $\nu_{\beta}$ is a Borel probability measure on $J_{\beta}$. In particular

$$
\left|\delta^{\beta} f(n)\right|^{2} \leq \int_{J_{\beta}}\left|\partial^{\beta} \tilde{f}(n+s)\right|^{2} d \nu_{\beta}(s)
$$

for all $n \in \mathbb{N}^{d_{1}}$. 
We give now a simplified version of the right-hand side of (17), in the case we restrict to the functional calculus of $L$ alone. In order to avoid issues of divergent series, it is however convenient at first to truncate the multiplier along the spectrum of $\mathbf{U}$.

Lemma 10. Let $\chi \in C_{c}^{\infty}(\mathbb{R})$ be supported in $[1 / 2,2], K \subseteq \mathbb{R}$ be compact and $M \in] 0, \infty\left[\right.$. If $F: \mathbb{R} \rightarrow \mathbb{C}$ is smooth and supported in $K$, and $F_{M}: \mathbb{R} \times \mathfrak{z}^{*} \rightarrow \mathbb{C}$ is given by

$$
F_{M}(\lambda, \eta)=F(\lambda) \chi(|\eta| / M)
$$

then, for all $r \in[0, \infty[$,

$$
\int_{G}\left\|\left.\left.u\right|^{r} \mathcal{K}_{F_{M}(L, \mathbf{U})}(z, u)\right|^{2} d z d u \leq C_{K, \chi, r} M^{d_{2}-2 r}\right\| F \|_{W_{2}^{r}}^{2}
$$

Proof. We may restrict to the case $r \in \mathbb{N}$, the other cases being recovered a posteriori by interpolation. Hence we need to prove that

$$
\int_{G}\left|u^{\alpha} \mathcal{K}_{F_{M}(L, \mathbf{U})}(z, u)\right|^{2} d z d u \leq C_{K, \chi, \alpha} M^{d_{2}-2|\alpha|}\|F\|_{W_{2}^{|\alpha|}}^{2}
$$

for all $\alpha \in \mathbb{N}^{d_{1}}$. On the other hand, if $m$ is defined by

$$
m(n, \mu, \eta)=F\left(\sum_{j=1}^{d_{1}} b_{j}^{\eta}\left\langle n_{j}\right\rangle_{j}+|\mu|_{\Sigma}\right) \chi(|\eta| / M),
$$

where $\langle\ell\rangle_{j}=2 \ell+r_{j}$ and $|\mu|_{\Sigma}=\sum_{j=1}^{\tilde{d_{1}}} \mu_{j}$, then the left-hand side of (19) is majorized by the right-hand side of (17), and we are reduced to prove

$$
\begin{aligned}
\sum_{n \in \mathbb{N}^{d_{1}}} \int_{\dot{\mathfrak{z}}} & \int_{\left[0, \infty\left[\tilde{d_{1}}\right.\right.}\left|\partial_{\eta}^{\gamma^{\iota}} \partial_{\mu}^{\theta^{\iota}} \delta^{\beta^{\iota}} m(n, \mu, \eta)\right|^{2}|\eta|^{2\left|\gamma^{\iota}\right|-2|\alpha|-2\left|\beta^{\iota}\right|+\left|a^{\iota}\right|+d_{1}} \\
& \times\left(1+n_{1}\right)^{a_{1}^{\iota}} \ldots\left(1+n_{d_{1}}\right)^{a_{d_{1}}^{\iota}} d \sigma_{\iota}(\mu) d \eta \leq C_{K, \chi, \alpha} M^{d_{2}-2|\alpha|}\|F\|_{W_{2}^{|\alpha|}}^{2}
\end{aligned}
$$

for all $\iota \in \tilde{I}_{\alpha}$, where $\tilde{I}_{\alpha}, \gamma^{\iota}, \theta^{\iota}, \beta^{\iota}, a^{\iota}, \sigma_{\iota}$ are as in Proposition 8 .

Note that the right-hand side of (20) makes sense for all $n \in \mathbb{R}^{d_{1}}$, and defines a smooth extension of $m$, which we still denote by $m$ by a slight abuse of notation. Hence, by Lemma 9,

$$
\left|\partial_{\eta}^{\gamma_{\iota}} \partial_{\mu}^{\theta^{\iota}} \delta^{\beta^{\iota}} m(n, \mu, \eta)\right|^{2} \leq \int_{J_{\iota}}\left|\partial_{\eta}^{\gamma^{\iota}} \partial_{\mu}^{\theta^{\iota}} \partial_{n}^{\beta^{\iota}} m(n+s, \mu, \eta)\right|^{2} d \nu_{\iota}(s)
$$

where $J_{\iota}=\prod_{j=1}^{d_{1}}\left[0, \beta_{j}^{\iota}\right]$ and $\nu_{\iota}$ is a suitable probability measure on $J_{\iota}$. Moreover the measure $\sigma_{\iota}$ in (21) is finite on compacta, and the right-hand side of (22) vanishes when $|\mu|_{\Sigma}>\max K$, because $\operatorname{supp} F \subseteq K$. Consequently (21) will be proved if we show that

$$
\begin{aligned}
\sum_{n \in \mathbb{N}^{d_{1}}} \int_{\dot{\mathfrak{z}}} \mid \partial_{\eta}^{\gamma^{\iota}} \partial_{\mu}^{\theta^{\iota}} & \left.\partial_{n}^{\beta^{\iota}} m(n+s, \mu, \eta)\right|^{2}|\eta|^{2\left|\gamma^{\iota}\right|-2|\alpha|-2\left|\beta^{\iota}\right|+\left|a^{\iota}\right|+d_{1}} \\
& \times\left(1+n_{1}\right)^{a_{1}^{\iota}} \ldots\left(1+n_{d_{1}}\right)^{a_{d_{1}}^{\iota}} d \eta \leq C_{K, \chi, \alpha} M^{d_{2}-2|\alpha|}\|F\|_{W_{2}^{|\alpha|}}^{2}
\end{aligned}
$$

for all $s \in J_{\iota}$ and $\mu \in[0, \max K]^{\tilde{d_{1}}}$, uniformly in $s$ and $\mu$.

As observed in the proof of Proposition 6, the $b_{j}^{\eta}$ are positive, smooth functions of $\eta \in \dot{\mathfrak{z}}$, homogeneous of degree 1 ; therefore, for all $n \in \mathbb{N}^{d_{1}}, j \in\left\{1, \ldots, d_{1}\right\}, \eta \in \dot{\mathfrak{z}}$, $s \in\left[0, \infty\left[{ }^{d_{1}}, \mu \in\left[0, \infty\left[^{\tilde{d}_{1}}\right.\right.\right.\right.$,

$$
|\eta|\left(1+n_{j}\right) \sim b_{j}^{\eta}\left\langle n_{j}\right\rangle_{j} \leq \sum_{l=1}^{d_{1}} b_{l}^{\eta}\left\langle n_{l}+s_{l}\right\rangle_{l}+|\mu|_{\Sigma},
$$


and the last quantity is bounded by the constant max $K$ whenever $(n+s, \mu, \eta) \in$ $\operatorname{supp} m$, because supp $F \subseteq K$. Hence the factors $|\eta|\left(1+n_{j}\right)$ in the left-hand side of (23) can be discarded, that is, we are reduced to proving (23) in the case $a^{\iota}=0$.

From (20) it is easily proved inductively that

$$
\begin{array}{r}
\partial_{\eta}^{\gamma^{\iota}} \partial_{\mu}^{\theta^{\iota}} \partial_{n}^{\beta^{\iota}} m(n, \mu, \eta)=\sum_{\substack{v \in \mathbb{N}^{d_{1}} \\
|v| \leq\left|\gamma^{\iota}\right|}} \sum_{q=0}^{\left|\gamma^{\iota}\right|-|v|} F^{\left(\left|\theta^{\iota}\right|+\left|\beta^{\iota}\right|+|v|\right)}\left(\sum_{j=1}^{d_{1}} b_{j}^{\eta}\left\langle n_{j}\right\rangle_{j}+|\mu|_{\Sigma}\right) \\
\times \Psi_{\iota, v, q}(\eta) M^{-q} \chi^{(q)}(|\eta| / M) \prod_{j=1}^{d_{1}}\langle n\rangle_{j}^{v_{j}}
\end{array}
$$

where $\Psi_{\iota, v, q}: \dot{\mathfrak{z}} \rightarrow \mathbb{R}$ is smooth and homogeneous of degree $\left|\beta^{\iota}\right|+|v|+q-\left|\gamma^{\iota}\right|$. By exploiting again (24) and the fact that $\operatorname{supp} F \subseteq K$, we than obtain that

$$
\begin{aligned}
\left|\partial_{\eta}^{\gamma^{\iota}} \partial_{\mu}^{\theta^{\iota}} \partial_{n}^{\beta^{\iota}} m(n, \mu, \eta)\right|^{2} \leq C_{K, \chi, \alpha} M^{2\left|\beta^{\iota}\right|-2\left|\gamma^{\iota}\right|} \tilde{\chi}(|\eta| / M) & \\
& \times \sum_{v=0}^{\left|\gamma^{\iota}\right|}\left|F^{\left(\left|\beta^{\iota}\right|+\left|\theta^{\iota}\right|+v\right)}\left(\sum_{j=1}^{d_{1}} b_{j}^{\eta}\left\langle n_{j}\right\rangle_{j}+|\mu|_{\Sigma}\right)\right|^{2},
\end{aligned}
$$

where $\tilde{\chi}$ is the characteristic function of $[1 / 2,2]$. Hence the left-hand side of (23), when $a^{\iota}=0$, is majorized by

$$
\begin{aligned}
& C_{K, \chi, \alpha} M^{d_{1}-2|\alpha|} \\
& \quad \times \sum_{v=0}^{\left|\gamma_{i}\right|} \int_{\dot{\mathfrak{z}}} \sum_{n \in \mathbb{N}^{d_{1}}}\left|F^{\left(\left|\beta^{\iota}\right|+\left|\theta^{\iota}\right|+v\right)}\left(\sum_{j=1}^{d_{1}} b_{j}^{\eta}\left\langle n_{j}+s_{j}\right\rangle_{j}+|\mu|_{\Sigma}\right)\right|^{2} \tilde{\chi}(|\eta| / M) d \eta .
\end{aligned}
$$

Let $S$ denote the unit sphere in $\mathfrak{z}^{*}$. By passing to polar coordinates and exploiting the homogeneity of the $b_{j}^{\eta}$, the integral in the above formula is majorized by

$$
\begin{array}{r}
C \int_{S} \int_{0}^{\infty} \sum_{n \in \mathbb{N}^{d_{1}}}\left|F^{\left(\left|\beta^{\iota}\right|+\left|\theta^{\iota}\right|+v\right)}\left(\rho \sum_{j=1}^{d_{1}} b_{j}^{\omega}\left\langle n_{j}+s_{j}\right\rangle_{j}+|\mu|_{\Sigma}\right)\right|^{2} \tilde{\chi}(\rho / M) \rho^{d_{2}} \frac{d \rho}{\rho} d \omega \\
\leq C M^{d_{2}} \int_{0}^{\infty}\left|F^{\left(\left|\beta^{\iota}\right|+\left|\theta^{\iota}\right|+v\right)}\left(\rho+|\mu|_{\Sigma}\right)\right|^{2} \int_{S} \sum_{n \in \mathbb{N}^{d_{1}}} \tilde{\chi}\left(\rho /\left(M\langle n\rangle_{\omega, s}\right)\right) d \omega \frac{d \rho}{\rho}
\end{array}
$$

where $\langle n\rangle_{\omega, s}=\sum_{j=1}^{d_{1}} b_{j}^{\omega}\left\langle n_{j}+s_{j}\right\rangle_{j} \sim 1+|n|$ uniformly in $\omega \in S$ and $s \in J_{\iota}$. Hence the sum in the right-hand side has at most $C_{\iota}(\rho / M)^{d_{1}}$ summands, and the integral on $S$ is majorized by $C_{\iota}(\rho / M)^{d_{1}}$. In conclusion, the left-hand side of (23) is majorized by

$$
\begin{aligned}
C_{K, \chi, \alpha} M^{d_{2}-2|\alpha|} \sum_{v=0}^{\left|\gamma_{i}\right|} \int_{0}^{\infty}\left|F^{\left(\left|\beta^{\iota}\right|+\left|\theta^{\iota}\right|+v\right)}\left(\rho+|\mu|_{\Sigma}\right)\right|^{2} & \rho^{d_{1}-1} d \rho \\
& \leq C_{K, \chi, \alpha} M^{d_{2}-2|\alpha|}\|F\|_{W_{2}^{|\alpha|}}^{2},
\end{aligned}
$$

because $d_{1} \geq 1$, supp $F \subseteq K$ and $\left|\beta^{\iota}\right|+\left|\theta^{\iota}\right|+\left|\gamma^{\iota}\right| \leq|\alpha|$, and we are done.

Proposition 11. Let $F: \mathbb{R} \rightarrow \mathbb{C}$ be smooth and such that $\operatorname{supp} F \subseteq K$ for some compact set $K \subseteq \mathbb{R}$. For all $r \in\left[0, d_{2} / 2[\right.$,

$$
\int_{G}\left|(1+|u|)^{r} \mathcal{K}_{F(L)}(z, u)\right|^{2} d z d u \leq C_{K, r}\|F\|_{W_{2}^{r}}^{2} .
$$


Proof. Take $\chi \in C_{c}^{\infty}(] 0, \infty[)$ such that $\operatorname{supp} \chi \subseteq[1 / 2,2]$ and $\sum_{k \in \mathbb{Z}} \chi\left(2^{-k} t\right)=1$ for all $t \in] 0, \infty$ [. If $F_{M}$ is defined for all $\left.M \in\right] 0, \infty\left[\right.$ as in Lemma [10, then $\mathcal{K}_{F_{M}(L, \mathbf{U})}$ is given by the right-hand side of (8), where $m$ is defined by (20), and moreover

$$
\sum_{j=1}^{d_{1}} b_{j}^{\eta}\left\langle n_{j}\right\rangle_{j}+|\mu|_{\Sigma} \geq C^{-1}|\eta|
$$

for all $\eta \in \dot{\mathfrak{z}}, \mu \in\left[0, \infty\left[^{\tilde{d}_{1}}\right.\right.$ and $n \in \mathbb{N}^{d_{1}}$, therefore $F_{M}(L, \mathbf{U})=0$ whenever $M>$ $2 C \max K$. Hence, if $k_{K} \in \mathbb{Z}$ is sufficiently large so that $2^{k_{K}}>2 C \max K$, then

$$
F(L)=\sum_{k \in \mathbb{Z}, k \leq k_{K}} F_{2^{k}}(L, \mathbf{U})
$$

(with convergence in the strong sense). Consequently an estimate for $\mathcal{K}_{F(L)}$ can be obtained, via Minkowski's inequality, by summing the corresponding estimates for $\mathcal{K}_{F_{2^{k}}}(L, \mathbf{U})$ given by Lemma 10. If $r<d / 2$, then the series $\sum_{k \leq k_{K}}\left(2^{k}\right)^{d_{2} / 2-r}$ converges, thus

$$
\left.\left.\int_{G}|| u\right|^{r} \mathcal{K}_{F(L)}(z, u)\right|^{2} d z d u \leq C_{K, r}\|F\|_{W_{2}^{r}}^{2}
$$

The conclusion follows by combining the last inequality with the corresponding one for $r=0$.

Let $|\cdot|_{\delta}$ be a $\delta_{t}$-homogeneous norm on $G$; take, e.g., $|(z, u)|_{\delta}=|z|+|u|^{1 / 2}$. Interpolation then allows us to improve the standard weighted estimate for a homogeneous sublaplacian on a stratified group.

Proposition 12. Let $F: \mathbb{R} \rightarrow \mathbb{C}$ be smooth and such that $\operatorname{supp} F \subseteq K$ for some compact set $K \subseteq \mathbb{R}$. For all $r \in\left[0, d_{2} / 2[, \alpha \geq 0\right.$ and $\beta>\alpha+r$,

$$
\int_{G}\left|\left(1+|(z, u)|_{\delta}\right)^{\alpha}(1+|u|)^{r} \mathcal{K}_{F(L)}(z, u)\right|^{2} d z d u \leq C_{K, \alpha, \beta, r}\|F\|_{W_{2}^{\beta}}^{2} .
$$

Proof. Note that $1+|u| \leq C\left(1+|(z, u)|_{\delta}\right)^{2}$. Hence, in the case $\alpha \geq 0, \beta>\alpha+2 r$, the inequality (25) follows by the mentioned standard estimate (see [16. Lemma 1.2] or [12, Theorem 2.7]). On the other hand, if $\alpha=0$ and $\beta \geq r$, then (25) is given by Proposition 11. The full range of $\alpha$ and $\beta$ is then obtained by interpolation.

We can finally prove the crucial estimate.

Proof of Proposition [3. Take $r \in](\operatorname{dim} G) / 2+d_{2} / 2-s, d_{2} / 2[$. Then

$$
s-r>(\operatorname{dim} G) / 2+d_{2} / 2-2 r=(\operatorname{dim} \mathfrak{v}) / 2+d_{2}-2 r,
$$

hence we can find $\alpha_{1}>(\operatorname{dim} \mathfrak{v}) / 2$ and $\alpha_{2}>d_{2}-2 r$ such that $s-r>\alpha_{1}+\alpha_{2}$. Set $w_{s}(z, u)=\left(1+|(z, u)|_{\delta}\right)^{\alpha}(1+|u|)^{r}$. The $L^{2}$ estimate (4) then follows from Proposition 12. On the other hand, for all $(z, u) \in G$,

$$
w_{s}^{-2}(z, u) \leq C_{s}(1+|z|)^{-2 \alpha_{1}}(1+|u|)^{-\alpha_{2}-2 r},
$$

and the right-hand side is integrable over $G \cong \mathfrak{v} \times \mathfrak{z}$ since $2 \alpha_{1}>\operatorname{dim} \mathfrak{v}$ and $\alpha_{2}+2 r>$ $d_{2}=\operatorname{dim} \mathfrak{z}$. Therefore $w_{s}^{-1} \in L^{2}(G)$, and The $L^{1}$ estimate (5) follows from (4) and Hölder's inequality.

\section{REMARKS ON THE VALIDITY OF THE ASSUMPTION AND DIRECT PRODUCTS}

In this section we do no longer suppose that $G$ and $L$ are a 2-step stratified Lie group and a sublaplacian satisfying Assumption (A).

As observed in $\$ 2$, a necessary condition for the validity of Assumption (A) is that the skewadjoint endomorphism $J_{\eta}$ of the first layer $\mathfrak{v}$ has constant rank for $\eta$ ranging in $\dot{\mathfrak{z}}=\mathfrak{z}^{*} \backslash\{0\}$. Here we show that this condition is also sufficient when the rank is minimal. 
Proposition 13. Let $G$ be a 2-step nilpotent Lie group, with Lie algebra $\mathfrak{g}=\mathfrak{v} \oplus \mathfrak{z}$, and let $\langle\cdot, \cdot\rangle$ be an inner product on $\mathfrak{v}$. Suppose that the skewadjoint endomorphism $J_{\eta}$ of $\mathfrak{v}$ has rank 2 for all $\eta \in \dot{\mathfrak{z}}$. Then $G$ satisfies Assumption (A) with the sublaplacian $L$ associated to the given inner product, and also with any other sublaplacian associated to an inner product on a complement of $\mathfrak{z}$.

Let moreover $G_{\mathbb{C}}$ be the complexification of $G$, considered as a real 2-step group, with Lie algebra $\mathfrak{g}_{\mathbb{C}}=\mathfrak{v}_{\mathbb{C}} \oplus \mathfrak{z} \mathbb{C}$, and let $\mathfrak{v}_{\mathbb{C}}$ be endowed with the real inner product induced by the inner product on $\mathfrak{v}$. Then $G_{\mathbb{C}}$, with the sublaplacian associated to the given inner product, satisfies Assumption (A).

Proof. From the normal form for skewadjoint endomorphisms, it follows immediately that, if $J_{\eta}$ has rank 2 , then $J_{\eta}^{2}$ has exactly one nonzero eigenvalue, and Assumption (A) is trivially verified. Moreover, if $\mathfrak{v}$ is identified with $\mathfrak{g} / \mathfrak{z}$, then ker $J_{\eta}$ corresponds to the subspace

$$
N_{\eta}=\left\{x+\mathfrak{z}: x \in \mathfrak{g} \text { and } \eta\left(\left[x, x^{\prime}\right]\right)=0 \text { for all } x^{\prime} \in \mathfrak{g}\right\}
$$

of $\mathfrak{g} / \mathfrak{z}$; hence the rank condition on $J_{\eta}$ can be rephrased by saying that $N_{\eta}$ has codimension 2 for all $\eta \in \dot{\mathfrak{z}}$, and this condition does not depend on the sublaplacian $L$ chosen on $G$.

Let $R\left(J_{\eta}\right)$ denote the range of $J_{\eta}$. We show now that, for all $\eta, \eta^{\prime} \in \dot{\mathfrak{z}}$, the intersection $R\left(J_{\eta}\right) \cap R\left(J_{\eta^{\prime}}\right)$ is nontrivial. If it were trivial, since $J_{\eta+\eta^{\prime}}=J_{\eta}+J_{\eta}^{\prime}$, we would have $\operatorname{ker} J_{\eta+\eta^{\prime}}=\operatorname{ker} J_{\eta} \cap \operatorname{ker} J_{\eta^{\prime}}$, hence

$$
R\left(J_{\eta+\eta^{\prime}}\right)=\left(\operatorname{ker} J_{\eta+\eta^{\prime}}\right)^{\perp}=R\left(J_{\eta}\right) \oplus R\left(J_{\eta^{\prime}}\right),
$$

thus $J_{\eta+\eta^{\prime}}$ would have rank 4 , contradiction.

Consider now the complexification $\mathfrak{g}_{\mathbb{C}}=\mathfrak{g} \oplus i \mathfrak{g}$. Via the linear identifications $\mathfrak{g}_{\mathbb{C}}=\mathfrak{g} \times \mathfrak{g}, \mathfrak{z}_{\mathbb{C}}^{*}=\mathfrak{z}^{*} \times \mathfrak{z}^{*}, \mathfrak{v}_{\mathbb{C}}=\mathfrak{v} \times \mathfrak{v}$, the skewsymmetric endomorphism $\tilde{J}_{\eta}$ of the first layer $\mathfrak{v}_{\mathbb{C}}$ corresponding to the element $\eta=\left(\eta_{R}, \eta_{I}\right) \in \mathfrak{z}_{\mathbb{C}}^{*}$ is given by

$$
\tilde{J}_{\eta}\left(x_{R}, x_{I}\right)=\left(J_{\eta_{R}} x_{R}+J_{\eta_{I}} x_{I}, J_{\eta I} x_{R}-J_{\eta_{R}} x_{I}\right) .
$$

Take now $\eta=\left(\eta_{R}, \eta_{I}\right) \in \dot{\mathfrak{z}} \mathbb{C}$; we want to show that $\tilde{J}_{\eta}^{2}$ has rank 4 and a unique nonzero eigenvalue. We distinguish several cases.

If $\eta_{I}=0$, then $\tilde{J}_{\eta}=J_{\eta_{R}} \times\left(-J_{\eta_{R}}\right)$, hence $\tilde{J}_{\eta}^{2}=J_{\eta_{R}}^{2} \times J_{\eta_{R}}^{2}$ satisfies the condition. The same argument gives the conclusion in the case $\eta_{R}=0$.

If both $\eta_{R}, \eta_{I} \in \dot{\mathfrak{z}}$, then $R\left(J_{\eta_{R}}\right) \cap R\left(J_{\eta_{I}}\right) \neq 0$, hence $\operatorname{dim}\left(R\left(J_{\eta_{R}}\right) \cap R\left(J_{\eta_{I}}\right)\right)$ is either 2 or 1 . In the first case, $R\left(J_{\eta_{R}}\right)=R\left(J_{\eta_{I}}\right)$, so $J_{\eta_{R}}$ and $J_{\eta_{I}}$ commute and (26) implies that

$$
\tilde{J}_{\eta}^{2}=\left(J_{\eta_{R}}^{2}+J_{\eta_{I}}^{2}\right) \times\left(J_{\eta_{R}}^{2}+J_{\eta_{I}}^{2}\right)
$$

since $J_{\eta_{R}}^{2}$ and $J_{\eta_{I}}^{2}$ are negative multiples of the same orthogonal projection, the conclusion follows.

Suppose now that $R\left(J_{\eta_{R}}\right) \cap R\left(J_{\eta_{I}}\right)=\mathbb{R} x$ for some unit vector $x \in \mathfrak{v}$, and set $y_{R}=J_{\eta_{R}} x, y_{I}=J_{\eta_{I}} x, b_{R}=\left|y_{R}\right|, b_{I}=\left|y_{I}\right|$; in particular $J_{\eta_{R}}^{2} x=-b_{R}^{2} x$ and $J_{\eta_{I}}^{2} x=-b_{I}^{2} x$. Since $J_{\eta_{R}}$ and $J_{\eta_{I}}$ are skewadjoint and of rank 2, necessarily $J_{\eta_{R}} x, J_{\eta_{I}} x \in x^{\perp}$ and $J_{\eta_{R}}\left(x^{\perp}\right)=J_{\eta_{I}}\left(x^{\perp}\right)=\mathbb{R} x$, therefore $J_{\eta_{R}} J_{\eta_{I}} x$ and $J_{\eta_{I}} J_{\eta_{R}} x$ are both multiples of $x$; on the other hand,

$$
\left\langle J_{\eta_{R}} J_{\eta_{I}} x, x\right\rangle=-\left\langle J_{\eta_{I}} x, J_{\eta_{R}} x\right\rangle=\left\langle x, J_{\eta_{I}} J_{\eta_{R}} x\right\rangle,
$$

hence $J_{\eta_{R}} J_{\eta_{I}} x=J_{\eta_{I}} J_{\eta_{R}} x$. This identity, together with (26), allows us easily to show that

$$
\begin{array}{cc}
\tilde{J}_{\eta}(x, 0)=\left(y_{R}, y_{I}\right), & \tilde{J}_{\eta}\left(y_{R}, y_{I}\right)=-\left(b_{R}^{2}+b_{I}^{2}\right)(x, 0), \\
\tilde{J}_{\eta}(0, x)=\left(y_{I},-y_{R}\right), & \tilde{J}_{\eta}\left(y_{I},-y_{R}\right)=-\left(b_{R}^{2}+b_{I}^{2}\right)(0, x) .
\end{array}
$$


Note that $b_{R}^{2}+b_{I}^{2}$ is the squared norm of both $\left(y_{R}, y_{I}\right)$ and $\left(y_{I},-y_{R}\right)$. Hence we would be done if we knew that $R\left(\tilde{J}_{\mu}\right)$ coincides with the linear span $W$ of $(x, 0)$, $(0, x),\left(y_{R}, y_{I}\right),\left(y_{I},-y_{R}\right)$.

In fact, we just need to show that $R\left(\tilde{J}_{\eta}\right)$ is contained in $W$, or equivalently, that $W^{\perp}$ is contained in ker $\tilde{J}_{\eta}$. On the other hand, if $v=\left(v_{R}, v_{I}\right) \in W^{\perp}$, then $v_{R}, v_{I} \in x^{\perp}$ and moreover

$$
\left\langle v_{R}, y_{R}\right\rangle+\left\langle v_{I}, y_{I}\right\rangle=0, \quad\left\langle v_{R}, y_{I}\right\rangle-\left\langle v_{I}, y_{R}\right\rangle=0
$$

hence $J_{\eta_{R}} v_{R}, J_{\eta_{R}} v_{I}, J_{\eta_{I}} v_{R}, J_{\eta_{I}} v_{I} \in \mathbb{R} x$, and

$$
\begin{aligned}
& \left\langle J_{\eta_{R}} v_{R}, x\right\rangle=-\left\langle v_{R}, y_{R}\right\rangle=\left\langle v_{I}, y_{I}\right\rangle=-\left\langle J_{\eta_{I}} v_{I}, x\right\rangle, \\
& \left\langle J_{\eta_{I}} v_{R}, x\right\rangle=-\left\langle v_{R}, y_{I}\right\rangle=-\left\langle v_{I}, y_{R}\right\rangle=\left\langle J_{\eta_{R}} v_{I}, x\right\rangle,
\end{aligned}
$$

therefore $J_{\eta_{R}} v_{R}=-J_{\eta_{I}} v_{I}$ and $J_{\eta_{I}} v_{R}=J_{\eta_{R}} v_{I}$, from which it follows immediately that $\tilde{J}_{\eta}\left(v_{R}, v_{I}\right)=0$.

The next proposition shows how groups and sublaplacians satisfying Assumption (A) may be "glued together", so to give a higher-dimensional group and a sublaplacian that satisfy Assumption (A) too.

Proposition 14. Suppose that, for $j=1,2$, the sublaplacian $L_{j}$ on the 2-step stratified Lie group $G_{j}$ satisfies Assumption (A). Suppose further that the centers of $G_{1}$ and $G_{2}$ have the same dimension. Let $G$ be the quotient of $G_{1} \times G_{2}$ given by any linear identification of the respective centers, and let $L=L_{1}^{\sharp}+L_{2}^{\sharp}$, where $L_{j}^{\sharp}$ is the pushforward of $L_{j}$ to $G$. Then the sublaplacian $L$ on the group $G$ satisfies Assumption (A).

Proof. Let $\mathfrak{g}_{j}$ be the Lie algebra of $G_{j}$, and let $\mathfrak{v}_{j}$ and $\langle\cdot, \cdot\rangle_{j}$ be the linear complement of the center $\mathfrak{z}_{j}$ and the inner product on $\mathfrak{v}_{j}$ determined by the sublaplacian $L_{j}$; denote moreover by $J_{j, \eta}$ the skewadjoint endomorphism of $\mathfrak{v}_{j}$ determined by $\eta \in \mathfrak{z}_{j}^{*}$.

The linear identification of the centers of $G_{1}$ and $G_{2}$ corresponds to a linear isomorphism $\phi: \mathfrak{z}_{1} \rightarrow \mathfrak{z}_{2}$, and the Lie algebra $\mathfrak{g}$ of the quotient $G$ can be identified with $\mathfrak{v}_{1} \times \mathfrak{v}_{2} \times \mathfrak{z}_{2}$, with Lie bracket

$$
\left[\left(v_{1}, v_{2}, z\right),\left(v_{1}^{\prime}, v_{2}^{\prime}, z^{\prime}\right)\right]=\left(0,0, \phi\left(\left[v_{1}, v_{1}^{\prime}\right]\right)+\left[v_{2}, v_{2}^{\prime}\right]\right) .
$$

Then the sublaplacian $L$ on $G$ corresponds to the inner product $\langle\cdot, \cdot\rangle$ on $\mathfrak{v}_{1} \times \mathfrak{v}_{2}$ defined by

$$
\left\langle\left(v_{1}, v_{2}\right),\left(v_{1}^{\prime}, v_{2}^{\prime}\right)\right\rangle=\left\langle v_{1}, v_{1}^{\prime}\right\rangle_{1}+\left\langle v_{2}, v_{2}^{\prime}\right\rangle_{2} .
$$

In particular, if $\phi^{*}: \mathfrak{z}_{2}^{*} \rightarrow \mathfrak{z}_{1}^{*}$ denotes the adjoint map of $\phi: \mathfrak{z}_{1} \rightarrow \mathfrak{z}_{2}$, then it is easily checked that the skewadjoint endomorphism of the first layer $\mathfrak{v}_{1} \times \mathfrak{v}_{2}$ of $\mathfrak{g}$ corresponding to an element $\eta$ of the dual $\mathfrak{z}_{2}^{*}$ of the center of $\mathfrak{g}$ is given by $J_{\eta}=J_{1, \phi^{*} \eta} \times J_{2, \eta}$. Hence the orthogonal decomposition of $\mathfrak{v}_{1} \times \mathfrak{v}_{2}$ giving the "simultaneous diagonalization" of the $J_{\eta}$ for all $\eta \in \dot{\mathfrak{z}}_{2}$ (in the sense of 92 ) is simply obtained by juxtaposing the corresponding orthogonal decompositions of $\mathfrak{v}_{1}$ and $\mathfrak{v}_{2}$.

Note that the direct product $G_{1} \times G_{2}$ itself need not satisfy Assumption (A), even if the factors $G_{1}$ and $G_{2}$ do. However a functional-analytic argument, as in [17, §4], can be used to deal with that case.

The key step in our proof of Theorem 2 is the weighted $L^{2}$ estimate (4) of Proposition 3. Let us now turn the conclusion of Proposition 3 into an assumption on a homogeneous sublaplacian $L$ on a stratified group $G$. 
Assumption $\left(\mathrm{B}_{t}\right)$. For all $s>t$ there exist a weight $w_{s}: G \rightarrow[1, \infty[$ such that $w_{s}^{-1} \in L^{2}(G)$ and, for all compact sets $K \subseteq \mathbb{R}$ and all Borel functions $F: \mathbb{R} \rightarrow \mathbb{C}$ with $\operatorname{supp} F \subseteq K$,

$$
\left\|w_{s} \mathcal{K}_{F(L)}\right\|_{L^{2}(G)} \leq C_{K, s}\|F\|_{W_{2}^{s}(\mathbb{R})} .
$$

Our Proposition 3 can then be rephrased by saying that Assumption (A) implies Assumption $\left(\mathrm{B}_{t}\right)$ for $t=(\operatorname{dim} G) / 2$. Note, on the other hand, that Assumption $\left(\mathrm{B}_{t}\right)$ makes sense for homogeneous sublaplacians on stratified groups $G$ of step other than 2. In fact, every homogeneous sublaplacian on a stratified group of homogeneous dimension $Q$ satisfies Assumption $\left(\mathrm{B}_{t}\right)$ for $t=Q / 2$, by [16, Lemma 1.2] (suitably extended so to admit multipliers that do not vanish in a neighborhood of the origin of $\mathbb{R}$; see, e.g., [17, Lemma 3.1] for the 1-dimensional case, and [12, Theorem 2.7] for the higher-dimensional case).

Differently from Assumption (A), the new Assumption $\left(\mathrm{B}_{t}\right)$ "behaves well" under direct products.

Proposition 15. For $j=1, \ldots, n$, let $L_{j}$ be a homogeneous sublaplacian on a stratified Lie group $G_{j}$ satisfying Assumption $\left(\mathrm{B}_{t_{j}}\right)$ for some $t_{j}>0$. Let $G=$ $G_{1} \times \cdots \times G_{n}$ and $L=L_{1}^{\sharp}+\cdots+L_{n}^{\sharp}$, where $L_{j}^{\sharp}$ is the pushforward to $G$ of the operator $L_{j}$. Then the sublaplacian $L$ on $G$ satisfies Assumption $\left(\mathrm{B}_{t}\right)$, where $t=t_{1}+\cdots+t_{n}$.

Proof. Take $s>t$. Then we can choose $s_{1}, \ldots, s_{n}$ such that $s_{1}>t_{1}, \ldots, s_{n}>t_{n}$ and $s=s_{1}+\cdots+s_{n}$. Let then $w_{j, s_{j}}: G_{j} \rightarrow[1, \infty[$ be the weight corresponding to $s_{j}$ given by Assumption $\left(\mathrm{B}_{t}\right)$ on $G_{j}$ and $L_{j}$, for $j=1, \ldots, n$. In particular $w_{j, s_{j}}^{-1} \in L^{2}\left(G_{j}\right)$ and, for all $\phi \in C_{c}^{\infty}(\mathbb{R})$, the map $F \mapsto \mathcal{K}_{(\phi F)\left(L_{j}\right)}$ is a bounded linear map of Hilbert spaces $W_{2}^{s_{j}}(\mathbb{R}) \rightarrow L^{2}\left(G_{j}, w_{j, s_{j}}^{2}\left(x_{j}\right) d x_{j}\right)$, where $d x_{j}$ denotes the Haar measure on $G_{j}$.

The operators $L_{1}^{\sharp}, \ldots, L_{n}^{\sharp}$ on $G$ are essentially self-adjoint and commute strongly, that is, they admit a joint spectral resolution and a joint functional calculus on $L^{2}(G)$, and moreover, for all bounded Borel functions $F_{1}, \ldots, F_{n}: \mathbb{R} \rightarrow \mathbb{C}$,

$$
\mathcal{K}_{\left(F_{1} \otimes \cdots \otimes F_{n}\right)\left(L_{1}^{\sharp}, \ldots, L_{n}^{\sharp}\right)}=\mathcal{K}_{F_{1}\left(L_{1}\right)} \otimes \cdots \otimes \mathcal{K}_{F_{n}\left(L_{n}\right)}
$$

11. Corollary 5.5]. Hence, for all $\phi_{1}, \ldots, \phi_{n} \in C_{c}^{\infty}(\mathbb{R})$, if $\phi=\phi_{1} \otimes \cdots \otimes \phi_{n}$, then the map $H \mapsto \mathcal{K}_{(\phi H)\left(L_{1}^{\sharp}, \ldots, L_{n}^{\sharp}\right)}$ is the tensor product of the maps $F_{j} \mapsto$ $\mathcal{K}_{\left(\phi_{j} F_{j}\right)\left(L_{j}\right)}$. Since these maps are bounded $W_{2}^{s_{j}}(\mathbb{R}) \rightarrow L^{2}\left(G_{j}, w_{j, s_{j}}^{2}\left(x_{j}\right) d x_{j}\right)$, the map $H \mapsto \mathcal{K}_{(\phi H)\left(L_{1}^{\sharp}, \ldots, F_{n}^{\sharp}\right)}$ is bounded $S_{2}^{\left(s_{1}, \ldots, s_{n}\right)} W\left(\mathbb{R}^{n}\right) \rightarrow L^{2}\left(G, w_{s}^{2}(x) d x\right)$, where $S_{2}^{\left(s_{1}, \ldots, s_{n}\right)} W\left(\mathbb{R}^{n}\right)=W_{2}^{s_{1}}(\mathbb{R}) \otimes \cdots \otimes W_{2}^{s_{n}}(\mathbb{R})$ is the $L^{2}$ Sobolev space with dominating mixed smoothness [20] of order $\left(s_{1}, \ldots, s_{n}\right)$, and $w_{s}=w_{1, s_{1}} \otimes \cdots \otimes w_{n, s_{n}}$ is the product weight on $G$. In particular, for all compact sets $K \subseteq \mathbb{R}$, if we choose the cutoffs $\phi_{j} \in C_{c}^{\infty}(\mathbb{R})$ so that $\left.\phi_{j}\right|_{K}=1$, then we deduce that, for all $H: \mathbb{R}^{n} \rightarrow \mathbb{C}$ with supp $H \subseteq K^{n}$,

$$
\left\|w_{s} \mathcal{K}_{H\left(L_{1}^{\sharp}, \ldots, L_{n}^{\sharp}\right)}\right\|_{L^{2}(G)} \leq C_{K, s}\|H\|_{S_{2}^{\left(s_{1}, \ldots, s_{n}\right)} W\left(\mathbb{R}^{n}\right)} .
$$

(cf. [12, Proposition 5.2]). Since

$$
\begin{aligned}
\|f\|_{S_{2}^{\left(s_{1}, \ldots, s_{n}\right)} W\left(\mathbb{R}^{n}\right)}^{2} & \sim \int_{\mathbb{R}^{n}}|\hat{f}(\xi)|^{2}\left(1+\left|\xi_{1}\right|\right)^{2 s_{1}} \ldots\left(1+\left|\xi_{n}\right|\right)^{2 s_{n}} d \xi \\
& \leq \int_{\mathbb{R}^{n}}|\hat{f}(\xi)|^{2}(1+|\xi|)^{2 s_{1}+\cdots+2 s_{n}} d \xi \sim\|f\|_{W_{2}^{s}\left(\mathbb{R}^{n}\right)}^{2},
\end{aligned}
$$

where $\hat{f}$ denotes the Euclidean Fourier transform of $f$, we see immediately that the estimate

$$
\left\|w_{s} \mathcal{K}_{H\left(L_{1}^{\sharp}, \ldots, L_{n}^{\sharp}\right)}\right\|_{L^{2}(G)} \leq C_{K, s_{1}, \ldots, s_{n}}\|H\|_{W_{2}^{s}\left(\mathbb{R}^{n}\right)},
$$


holds true whenever $K \subseteq \mathbb{R}$ is compact and $H: \mathbb{R}^{n} \rightarrow \mathbb{C}$ is supported in $K^{n}$.

Take now a compact set $K \subseteq \mathbb{R}$ and choose a smooth cutoff $\eta_{K} \in C_{c}^{\infty}(\mathbb{R})$ such that $\left.\eta_{K}\right|_{[0, \max K]}=1$. Let $F: \mathbb{R} \rightarrow \mathbb{C}$ be such that $\operatorname{supp} F \subseteq K$, and define $H: \mathbb{R}^{n} \rightarrow \mathbb{C}$ by

$$
H\left(\lambda_{1}, \ldots, \lambda_{n}\right)=F\left(\lambda_{1}+\cdots+\lambda_{n}\right) \eta_{K}\left(\lambda_{1}\right) \ldots \eta_{K}\left(\lambda_{n}\right)
$$

for all $\left(\lambda_{1}, \ldots, \lambda_{n}\right) \in \mathbb{R}^{n}$. Then $\operatorname{supp} H \subseteq\left(\operatorname{supp} \eta_{K}\right)^{n}$, and

$$
F\left(\lambda_{1}+\cdots+\lambda_{n}\right)=H\left(\lambda_{1}, \ldots, \lambda_{n}\right)
$$

for all $\left(\lambda_{1}, \ldots, \lambda_{n}\right) \in\left[0, \infty\left[^{n}\right.\right.$. Since the operators $L_{1}, \ldots, L_{n}$ are nonnegative, the joint spectrum of $L_{1}^{\sharp}, \ldots, L_{n}^{\sharp}$ is contained in $\left[0, \infty\left[^{n}\right.\right.$, hence

$$
F(L)=F\left(L_{1}^{\sharp}+\cdots+L_{n}^{\sharp}\right)=H\left(L_{1}^{\sharp}, \ldots, L_{n}^{\sharp}\right) .
$$

Consequently, by (28) and the smoothness of the map $\left(\lambda_{1}, \ldots, \lambda_{n}\right) \mapsto \lambda_{1}+\cdots+\lambda_{n}$ we obtain that

$$
\left\|w_{s} \mathcal{K}_{F(L)}\right\|_{L^{2}(G)} \leq C_{K, s}\|H\|_{W_{2}^{s}\left(\mathbb{R}^{n}\right)} \leq C_{K, s}\|F\|_{W_{2}^{s}(\mathbb{R})} .
$$

Since clearly $w_{s}^{-1}=w_{1, s_{1}}^{-1} \otimes \cdots \otimes w_{n, s_{n}}^{-1} \in L^{2}(G)$, we are done.

The previous results, together with the known weighted estimates for abelian [17. Lemma 3.1] and Métivier [8, 9, 12] groups, then yield the following extension of Theorem 2

Theorem 16. For $j=1, \ldots, n$, suppose that $L_{j}$ is a homogeneous sublaplacian on a stratified Lie group $G_{j}$. Suppose further that, for each $j \in\{1, \ldots, n\}$, at least one of the following conditions holds:

- $G_{j}$ and $L_{j}$ satisfy Assumption (A);

- $G_{j}$ is a Métivier group;

- $G_{j}$ is abelian.

Let $G=G_{1} \times \cdots \times G_{n}$ and $L=L_{1}^{\sharp}+\cdots+L_{n}^{\sharp}$, as in Proposition 15. If $F: \mathbb{R} \rightarrow \mathbb{C}$ satisfies

$$
\|F\|_{M W_{2}^{s}}<\infty
$$

for some $s>(\operatorname{dim} G) / 2$, then $F(L)$ is of weak type $(1,1)$ and bounded on $L^{p}(G)$ for all $p \in] 1, \infty[$.

\section{ACKNOWLedgments}

I am deeply grateful to Detlef Müller for stimulating discussions and his continuous encouragement. I am also grateful for the opportunity of visiting the Mathematical Institute of the University of Wrocław in the first bimester of 2011; numerous discussions, particularly with Jacek Dziubański and Waldemar Hebisch, have greatly contributed to my understanding of problems related to spectral multipliers.

\section{REFERENCES}

[1] F. Astengo, M. Cowling, B. Di Blasio, and M. Sundari, Hardy's uncertainty principle on certain Lie groups, J. London Math. Soc. (2) 62 (2000), no. 2, 461-472.

[2] F. Astengo, B. Di Blasio, and F. Ricci, Gelfand pairs on the Heisenberg group and Schwartz functions, J. Funct. Anal. 256 (2009), no. 5, 1565-1587.

[3] M. Christ, $L^{p}$ bounds for spectral multipliers on nilpotent groups, Trans. Amer. Math. Soc. 328 (1991), no. 1, 73-81.

[4] L. De Michele and G. Mauceri, $L^{p}$ multipliers on the Heisenberg group, Michigan Math. J. 26 (1979), no. 3, 361-371.

[5] A. Erdélyi, W. Magnus, F. Oberhettinger, and F. G. Tricomi, Higher transcendental functions. Vol. II, Robert E. Krieger Publishing Co. Inc., Melbourne, Fla.

[6] G. B. Folland and E. M. Stein, Hardy spaces on homogeneous groups, Mathematical Notes, vol. 28, Princeton University Press, Princeton, N.J., 1982. 
[7] G. B. Folland, Harmonic analysis in phase space, Annals of Mathematics Studies, vol. 122, Princeton University Press, Princeton, NJ, 1989.

[8] W. Hebisch, Multiplier theorem on generalized Heisenberg groups, Colloq. Math. 65 (1993), no. 2, 231-239.

[9] W. Hebisch and J. Zienkiewicz, Multiplier theorem on generalized Heisenberg groups. II, Colloq. Math. 69 (1995), no. 1, 29-36.

[10] A. Martini, Algebras of differential operators on Lie groups and spectral multipliers, Tesi di perfezionamento (PhD thesis), Scuola Normale Superiore, Pisa, 2010, arXiv:1007.1119.

[11] A. Martini, Spectral theory for commutative algebras of differential operators on Lie groups, J. Funct. Anal. 260 (2011), no. 9, 2767-2814.

[12] - Analysis of joint spectral multipliers on Lie groups of polynomial growth, Ann. Inst. Fourier (Grenoble) 62 (2012), no. 4, 1215-1263.

[13] A. Martini and D. Müller, $L^{p}$ spectral multipliers on the free group $N_{3,2}$, (2012), Preprint. arXiv: 1210.8090.

[14] - A sharp multiplier theorem for Grushin operators in arbitrary dimensions, (2012), Preprint. arXiv:1210.3564.

[15] A. Martini and A. Sikora, Weighted Plancherel estimates and sharp spectral multipliers for the Grushin operators, (2012), To appear in Math. Res. Lett. arXiv:1204.1159.

[16] G. Mauceri and S. Meda, Vector-valued multipliers on stratified groups, Rev. Mat. Iberoamericana 6 (1990), no. 3-4, 141-154.

[17] D. Müller and E. M. Stein, On spectral multipliers for Heisenberg and related groups, J. Math. Pures Appl. (9) 73 (1994), no. 4, 413-440.

[18] D. Müller, A restriction theorem for the Heisenberg group, Ann. of Math. (2) 131 (1990), no. 3, 567-587.

[19] D. Müller, F. Ricci, and E. M. Stein, Marcinkiewicz multipliers and multi-parameter structure on Heisenberg (-type) groups. II, Math. Z. 221 (1996), no. 2, 267-291.

[20] H.-J. Schmeisser, Recent developments in the theory of function spaces with dominating mixed smoothness, Nonlinear Analysis, Function Spaces and Applications. Proceedings of the Spring School held in Prague, May 30-June 6, 2006, vol. 8, Czech Academy of Sciences, Mathematical Institute, Praha, 2007, pp. 145-204.

[21] S. Thangavelu, Lectures on Hermite and Laguerre expansions, Mathematical Notes, vol. 42, Princeton University Press, Princeton, NJ, 1993.

[22] J. F. Torres Lopera, The cohomology and geometry of Heisenberg-Reiter nilmanifolds, Differential geometry, Peñíscola 1985, Lecture Notes in Math., vol. 1209, Springer, Berlin, 1986, pp. 292-301.

Alessio Martini, Mathematisches Seminar, Christian-Albrechts-Universität Zu Kiel, Ludewig-Meyn-Str. 4, D-24118 Kiel, Germany

E-mail address: martini@math.uni-kiel.de 